\title{
PRINCIPE VARIATIONNEL ET GROUPES KLEINIENS
}

\author{
Jean-Pierre Otal et Marc Peigné
}

Summary - Let $\Gamma$ be a non-elementary Kleinian group acting on a Cartan-Hadamard manifold $\tilde{X}$; denote by $\Lambda(\Gamma)$ the non-wandering set of the geodesic flow $\left(\phi_{t}\right)$ acting on the unit tangent bundle $T^{1}(\tilde{X} / \Gamma)$. When $\Gamma$ is convex cocompact (i.e. $\Lambda(\Gamma)$ is compact), the restriction of $\left(\phi_{t}\right)$ to $\Lambda(\Gamma)$ is an Axiom A flow : therefore, by a theorem of Bowen-Ruelle, there exists a unique invariant measure on $\Lambda(\Gamma)$ which has maximal entropy. In this paper, we study the case of an arbitrary Kleinian group $\Gamma$. We show that there exists a measure of maximal entropy for the restriction of $\left(\phi_{t}\right)$ to $\Lambda(\Gamma)$ if and only if the Patterson-Sullivan measure is finite ; furthermore when this measure is finite, it is the unique measure of maximal entropy.

By a theorem of Handel-Kitchens, the supremum of the measure-theoretic entropies equals the infimum of the entropies of the distances $d$ on $\Lambda(X)$; when $\Gamma$ is geometrically finite, we show that this infimum is achieved by the Riemannian distance $d$ on $\Lambda(X)$.

\section{Classification A.M.S :}

Primary 37C40, 37D40, 37B40, , 37D35

Secondary 28A50

Dans cet article, $\tilde{X}$ désignera une variété riemannienne complète simplement connexe, dont les courbures sectionnelles sont comprises entre deux constantes négatives $-\alpha^{2}$ et $-\beta^{2}(0<\alpha \leq \beta)$. Un groupe Kleinien sera un groupe discret d'isométries de $\tilde{X}$, non-élémentaire (i.e. qui ne possède pas de sous-groupes abéliens d'indice fini) et sans torsion : un groupe Kleinien $\Gamma$ opère sans points fixes sur $\tilde{X}$ et on note $X=\tilde{X} / \Gamma$ la variété riemannienne quotient. L'ensemble non-errant du flot géodésique $\left(\phi_{t}\right)$ agissant sur le fibré unitaire $T^{1} X$ est noté $\Lambda(\Gamma)$ : c'est sur cet ensemble que se concentre la dynamique intéressante du flot.

Nous allons relier certains invariants de la restriction de $\left(\phi_{t}\right)$ à $\Lambda(\Gamma)$ à l'exposant critique $d u$ groupe $\Gamma$, un invariant de $\Gamma$. Ce nombre $\delta(\Gamma)$ est défini comme l'exposant critique de la série, dite de Poincaré, $\sum_{\gamma \in \Gamma} e^{-s d(o, \gamma o)}$, où $o$ est un point de $\tilde{X}$; en d'autres termes, $\delta(\Gamma)=$ $\limsup _{r \rightarrow \infty} \frac{1}{r} \log \operatorname{card}\{\gamma / d(o, \gamma o) \leq r\}$.

Jean-Pierre Otal UMPA, UMR 128 CNRS,ENS Lyon, 46 allée d'Italie, 69364 Lyon Cedex 7 et MAPMO, Université d'Orléans, Rue de Chartres, B. P. 6759, 45067 Orléans cedex 2

Marc Peigné, LMPT, UMR 6083, Faculté des sciences et techniques, Parc de Grandmont, 37200 Tours

Typeset by $\mathcal{A} \mathcal{M S}-\mathrm{TEX}_{\mathrm{E}}$ 
Soit $\sigma=\left(\sigma_{x}\right)_{x \in \tilde{X}}$ une famille de mesures de Patterson sur $\partial X$; rappelons qu'en général, une telle famille n'est pas unique, mais que lorsque $\Gamma$ est de type divergent, c'est-à-dire lorsque la série de Poincaré ci-dessus diverge pour $s=\delta(\Gamma)$, elle l'est. Nous rappellerons dans $\S 3$ la construction importante de Sullivan qui permet d'associer à la famille $\left(\sigma_{x}\right)_{x \in \tilde{X}}$ une mesure de Radon $\mu$ invariante par le flot $\left(\phi_{t}\right)$ et de support $\Lambda(\Gamma)$. Si cette mesure est finie, on la supposera toujours normalisée en une mesure de probabilité.

Lorsque $\Gamma$ est convexe cocompact - c'est-à-dire lorsque $\Lambda(\Gamma)$ est compact -, la mesure $\mu$ est finie et son entropie égale à $\delta(\Gamma)$; de plus, l'entropie topologique du flot géodésique sur $\Lambda(\Gamma)$ vaut aussi $\delta(\Gamma)$ (voir [Su2]). En fait, le flot $\left(\phi_{t}\right)$ restreint à $\Lambda(\Gamma)$ est Axiome A : par un résultat de Bowen et Ruelle [BR], on sait alors que $\mu$ est l'unique mesure d'entropie maximale.

Ce sont ces problèmes que nous étudierons, mais pour des groupes $\Gamma$ qui ne sont plus convexes cocompacts : calculer l'entropie de la mesure $\mu$, calculer l'entropie topologique de $\left(\phi_{t}\right)$ restreint à $\Lambda(\Gamma)$, et décider s'il existe une unique mesure qui maximise l'entropie.

Si l'on s'intéresse, lorsque $\Gamma$ n'est plus convexe cocompact, à l'entropie topologique du flot $\left(\phi_{t}\right)$ restreint à $\Lambda(\Gamma)$, il faut tenir compte du fait que l'espace $\Lambda(\Gamma)$ du flot n'est pas compact. Pour un flot sur un espace métrique qui n'est pas compact, Bowen a précisé la notion d'entropie topologique [Bowe]. Cette entropie $h_{d}$ du flot $\left(\phi_{t}\right)$ restreint à $\Lambda(\Gamma)$ dépend donc du choix d'une métrique $d$ sur $\Lambda(\Gamma)$ et nous l'appellerons l'entropie de la distance $d$; l'entropie topologique $h_{\text {top }} d u$ flot $\left(\phi_{t}\right)$ est ensuite définie comme l'infimum des entropies $h_{d}$ sur l'ensemble de toutes les distances $d$ sur $\Lambda(\Gamma)$ qui induisent la topologie usuelle. C'est pour cette entropie topologique que l'on a un Principe variationnel, comme dans le cas d'un flot sur un espace compact : en effet, Handel et Kitchens ont montré que l'entropie topologique $h_{\text {top }}$ est la borne supérieure des entropies des mesures finies invariantes $[\mathrm{HK}]$. Dans cet article, nous calculerons l'entropie topologique $h_{\text {top }}$ pour un groupe Kleinien $\Gamma$ quelconque et étudierons la question de l'existence d'une mesure d'entropie maximale.

Théorème 1. Soit $\Gamma$ un groupe Kleinien d'isométries de $\tilde{X}$. Alors $h_{\mathrm{top}}=\delta(\Gamma) ;$ de plus, il existe une mesure de probabilité invariante $m$ qui maximise l'entropie si et seulement si la mesure de Patterson-Sullivan est finie et dans ce cas $m=\mu$.

La première généralisation de la notion de groupe cocompact est celle de groupe géométriquement fini. La définition et les propriétés de ces groupes seront rappelées dans $\S 1$ mais disons déjà que cette famille inclut tous les groupes de covolume fini, c'est-à-dire ceux tels que $X=\tilde{X} / \Gamma$ est une variété de volume fini. Lorsque $\Gamma$ est géométriquement fini, le domaine de la dynamique de $\left(\phi_{t}\right)$, c'est-à-dire l'ensemble non-errant $\Lambda(\Gamma)$, même s'il n'est pas forcément compact, est toujours la réunion d'un compact et d'un nombre fini de bouts dont la géométrie est bien comprise [Bowd]. Ces groupes ont été considérés par D. Sullivan dans [Su2] où il est montré que pour un groupe géométriquement fini $\Gamma$ d'isométries de l'espace hyperbolique réel $\mathbb{H}^{n}$, la mesure $\mu$ est finie et son entropie est égale à $\delta(\Gamma)$. Pour les groupes géométriquement finis d'isométries d'un espace symétrique de rang 1 , Corlette et Iozzi ont aussi montré la finitude de $\mu$ (voir [CI]).

Mais pour un groupe Kleinien $\Gamma$ agissant sur un espace $\tilde{X}$ général, la mesure $\mu$ n'est pas toujours finie. Lorsque $\Gamma$ est géométriquement fini, il existe un critère "local" portant sur les sous-groupes paraboliques de $\Gamma$ pour que ce soit le cas et on peut construire des groupes géométriquement finis pour lesquels $\mu$ est infinie $[\mathrm{DOP}]$; il existe aussi des groupes Kleiniens qui ne sont pas géométriquement finis, mais tels que $\mu$ est finie (voir $[\mathrm{A}],[\mathrm{Pe}]$ ).

La démonstration du Théorème 1 comprend deux parties, une partie de théorie de la mesure et une partie de nature géométrique. Nous montrerons d'abord :

Théorème 2. Soit $\Gamma$ un groupe Kleinien d'isométries de $\tilde{X}$. Alors,

(1) l'entropie de toute mesure de probabilité $\left(\phi_{t}\right)$-invariante est inférieure à $\delta(\Gamma)$; 
(2) si la mesure de Patterson-Sullivan est finie, son entropie vaut $\delta(\Gamma)$ et c'est l'unique mesure de probabilité d'entropie $\delta(\Gamma)$;

(3) si la mesure de Patterson-Sullivan est infinie, il n'existe pas de mesures de probabilité d'entropie $\delta(\Gamma)$.

La démonstration de ce Théorème utilisera de manière essentielle la théorie des partitions mesurables de Rokhlin et nous en rappellerons d'abord quelques propriétés dans $\S 2$. Les trois points du Théorème 2 sont démontrés simultanément; la méthode est à rapprocher de celle du Théorème de Ledrappier et Young qui caractérise les mesures, invariantes par un difféomorphisme d'une variété compacte, et pour lesquelles on a égalité dans la formule de Pesin. Comme dans [LY], on se fixe une mesure de probabilité invariante et ergodique $m$ et on commence par construire une partition $m$-mesurable $\xi$, d'entropie égale à $h_{m}(\phi)$ et dont les atomes sont contenus dans les feuilles du feuilletage fortement instable. Lorsque $m$ est la mesure de Patterson-Sullivan $\mu$, supposée finie, la formule de Rokhlin donne alors directement que l'entropie de $\mu$ vaut $\delta(\Gamma)$; l'inégalité de Jensen entraîne ensuite qu'une mesure d'entropie $\delta(\Gamma)$ ne peut-être que $\mu$. Même si l'existence de ces partitions est déjà établie dans [LS] et [LY], et dans un cadre plus général, nous la redonnerons dans la section $\S 2$, car leur construction dans notre cas particulier se simplifie. Le Théorème 2 sera ensuite démontré dans $\S 3$.

Un fois admis le Théorème 2 , la démonstration du Théorème 1 se ramène à voir que $h_{\text {top }} \geq \delta(\Gamma)$. En effet, si $h_{\text {top }}$ était strictement supérieur à $\delta(\Gamma)$, il existerait d'après le Principe variationnel de Handel et Kitchens des mesures finies dont l'entropie est arbitrairement proche de $h_{\text {top }}$, et en particulier strictement supérieure à $\delta(\Gamma)$; ceci est impossible d'après le Théorème 2 . La deuxième partie du Théorème 1 est alors contenue dans le Théorème 2. On est donc ramené à montrer que, pour toute distance $d^{\prime}$ sur $\Lambda(\Gamma)$, l'entropie $h_{d^{\prime}}$ vérifie $h_{d^{\prime}} \geq \delta(\Gamma)$ : nous verrons que ce résultat est à rapprocher du théorème de Bishop et Jones qui identifie la dimension de Hausdorff de l'ensemble limite radial d'un groupe Kleinien dans $\operatorname{PSL}(2, \mathbb{C})$ avec l'exposant critique $\delta(\Gamma)[\mathrm{BJ}]$ et les démonstrations sont vraiment parallèles. C'est la partie géométrique du Théorème 1 et elle sera traîtée dans la section $\S 4$.

Toutefois, la distance la plus "naturelle" sur le fibré tangent unitaire $T^{1} X$ est la distance riemannienne et on peut se poser la question du calcul de son l'entropie métrique. Ce calcul semble difficile dans la situation d'un groupe Kleinien $\Gamma$ quelconque. Dans la section $\S 4$, nous démontrerons le résultat suivant :

Proposition 3. Soit $\Gamma$ un groupe géométriquement fini d'isométries de $\tilde{X}$. Alors, l'entropie du flot $\left(\phi_{t}\right)$ restreint à $\Lambda(\Gamma)$ pour la distance riemannienne d est égale à $\delta(\Gamma)$.

L'inégalité $h_{d} \geq \delta(\Gamma)$ est vraie pour tous les groupes $\Gamma$, d'après le Théorème 1 . Par contre, pour établir l'inégalité $h_{d} \leq \delta(\Gamma)$, nous utiliserons de manière essentielle la description géométrique des bouts du cœur de Nielsen de la variété quotient $\tilde{X} / \Gamma$ lorsque $\Gamma$ est géométriquement fini. Chacun de ces bouts est quasi-isométrique à une demi-droite : l'idée de la démonstration est alors que l'entropie topologique du flot "restreint" à un tel bout est nulle.

Notons que pour un groupe Kleinien $\Gamma$ quelconque, la Proposition 3 devient fausse. En effet, si $\Gamma$ et $\Gamma^{\prime}$ sont deux groupes Kleiniens tels que $\Gamma \subset \Gamma^{\prime}$ et $L\left(\Gamma^{\prime}\right)=L(\Gamma)$, alors l'entropie métrique du flot géodésique sur $\Lambda(\Gamma)$ pour la distance riemannienne $d$ est supérieure à celle du flot géodésique sur $\Lambda\left(\Gamma^{\prime}\right)$ (toujours pour la distance riemannienne). Ceci découle directement de la définition de l'entropie $h_{d}\left(\right.$ cf. $\S 4$ ) et du fait que la projection de revêtement $\tilde{X} / \Gamma \rightarrow \tilde{X} / \Gamma^{\prime}$ induit un revêtement de $\Lambda(\Gamma) \rightarrow \Lambda\left(\Gamma^{\prime}\right)$ qui décroît la distance riemannienne. Maintenant l'égalité des ensembles limites $L(\Gamma)=L\left(\Gamma^{\prime}\right)$ a lieu dès que $\Gamma$ est un sous-groupe distingué non-trivial, par exemple le noyau d'un homomorphisme non-trivial de $\Gamma^{\prime}$ sur un groupe $G$. Or, on peut construire des exemples de 
groupes $\Gamma^{\prime}$ qui admettent un homomorphisme $\psi: \Gamma^{\prime} \rightarrow G$ tels que $\delta(\operatorname{Ker} \psi)<\delta\left(\Gamma^{\prime}\right)$ (cf. [K2]). Une question naturelle est donc la suivante:

Question. Soit $\Gamma$ un groupe Kleinien. Existe-t'il une distance $d$ sur $\Lambda(\Gamma)$ telle que $h_{d}=h_{\text {top }}$ ?

Dans tout cet article, $\pi$ désignera la projection canonique du fibré unitaire $T^{1} X$ sur $X, \tilde{\pi}$ celle de $T^{1} \tilde{X}$ sur $\tilde{X}$. La distance riemanienne dans $\tilde{X}$ sera notée $d_{\tilde{X}}$.

Nous remercions les referees pour les nombreuses remarques qu'ils ont faites et qui ont permis d'améliorer la rédaction de cet article. Nous tenons aussi à remercier ici F. Ledrappier pour les discussions fructueuses que nous avons eues avec lui sur la théorie de l'entropie.

\section{$\S 1$. Rappels sur les groupes Kleiniens et sur les mesures de Patterson-Sullivan.}

- Groupes Kleiniens.

On note $\partial \tilde{X}$ le bord visuel de $\tilde{X}$. Tout vecteur $v$ de $T^{1} \tilde{X}$ détermine de manière unique une géodésique notée $\left.v_{s}:\right]-\infty, \infty\left[\rightarrow \tilde{X}\right.$ de condition initiale $v_{0}=v$. On notera $v^{+}$et $v^{-}$les deux bouts de cette géodésique dans $\partial \tilde{X}$.

Étant donné un vecteur $\tilde{v} \in T^{1} \tilde{X}$, on note $W^{+}(\tilde{v})\left(\right.$ resp. $\left.W^{-}(\tilde{v})\right)$ la variété fortement instable (resp. fortement stable) de $\tilde{v}$ pour le flot géodésique sur $T^{1} \tilde{X}: W^{+}(\tilde{v})\left(\right.$ resp. $W^{-}(\tilde{v})$ ) est l'ensemble des vecteurs sortant (resp. rentrant) normaux à l'horosphère passant par le point $\tilde{\pi}(\tilde{v})$ et centrée au point $\tilde{v}^{+}$. Étant donné un vecteur $v \in T^{1} X$, on note $W^{+}(v)\left(\right.$ resp. $W^{-}(v)$ ) la variété fortement instable (resp. fortement stable) de $v$ pour le flot $\left(\phi_{t}\right)$ : c'est exactement l'image de $W^{ \pm}(\tilde{v})$ par la projection de revêtement $T^{1} \tilde{X} \rightarrow T^{1} X$. Le feuilletage instable $W^{+}$(resp. le feuilletage instable $W^{-}$) est défini par la partition de $T^{1} X$ en les feuilles $W^{+}(v)$ (resp. en les feuilles $W^{-}(v)$ ).

Si $\Gamma$ est un groupe Kleinien d'isométries de $\tilde{X}$, l'ensemble limite de $\Gamma$ est le plus petit fermé non-vide $L(\Gamma)$ de $\partial \tilde{X}$ qui est invariant par l'action de $\Gamma$. L'ensemble non-errant du flot géodésique $\left(\phi_{t}\right)$ sur $T^{1} X$, noté $\Lambda(\Gamma)$, est le projeté sur $T^{1} X$ de l'ensemble des vecteurs de $T^{1} \tilde{X}$ dont les bouts $v^{+}$et $v^{-}$sont dans l'ensemble limite $L(\Gamma)$.

On note $C(\Gamma)$ l'enveloppe convexe de $L(\Gamma)$ dans $\tilde{X} \cup \partial \tilde{X}$ : c'est un fermé invariant par $\Gamma$ et l'espace quotient $N(\Gamma)=C(\Gamma) / \Gamma$ est appelé le cœur de Nielsen de $X$. Par définition, $\Lambda(\Gamma)$ est contenu dans la préimage $\pi^{-1}(N(\Gamma))$.

Rappelons quelques propriétés des groupes géométriquement finis que nous utiliserons dans la section $\S 4$ (cf. [Bowd] pour un exposé détaillé). On dit qu'un groupe Kleinien $\Gamma$ est géométriquement fini lorsque, pour $\epsilon>0$, l' $\epsilon$-voisinage $N_{\epsilon}(\Gamma)$ de $N(\Gamma)$ est de volume fini. Le cœur de Nielsen $N(\Gamma)$ d'un groupe géométriquement fini se décompose en la réunion disjointe d'un compact $C_{0}$ et d'une famille finie $\left\{C_{1}, \ldots, C_{l}\right\}$ de "bouts cuspidaux" : pour $i \geq 1$, chaque $C_{i}$ est isométrique au quotient de l'intersection de $C(\Gamma)$ et d'une horoboule $\tilde{\mathcal{H}}_{i}$ par un sous-groupe parabolique maximal $\mathcal{P}_{i} \subset \Gamma$. Notons $\partial \tilde{\mathcal{H}}_{i}$ l'horosphère, bordant $\tilde{\mathcal{H}}_{i}$ et paramétrons $\tilde{\mathcal{H}}_{i}$ par le produit $\partial \tilde{\mathcal{H}}_{i} \times\left[0, \infty\left[\right.\right.$, en associant au point $p \in \tilde{\mathcal{H}}_{i}$ le couple $(h, t)$ où $h$ est la projection de $p$ sur l'horosphère $\tilde{\mathcal{H}}_{i}$ et $t$ la distance algébrique de $p$ à cette horosphère. Ce paramétrage est invariant par rapport aux isométries de $\mathcal{P}_{i}$. Il induit un paramétrage de $\mathcal{H}_{i}=\tilde{\mathcal{H}}_{i} / \mathcal{P}_{i}$ par le produit $\partial \mathcal{H}_{i} \times[0, \infty[$. Lorsque $\Gamma$ est géométriquement fini, on sait que l'intersection du cœur de Nielsen $N(\Gamma)$ avec $\mathcal{H}_{i}$ est contenue dans le produit $N_{i} \times\left[0, \infty\left[\right.\right.$, pour un certain compact $N_{i} \subset \partial \mathcal{H}_{i}$; de plus, le diamètre de l'intersection $N_{i}^{r}=N(\Gamma) \cap\left(\partial \mathcal{H}_{i} \times\{r\}\right)$ tend vers 0 quand $r$ tend vers $\infty$.

- Mesures de Patterson-Sullivan.

Rappellons la construction fondamentale de Patterson. Soit $\Gamma$ un groupe Kleinien et soit $x \in \tilde{X}$. On peut choisir une fonction $h: \mathbb{R}^{+} \rightarrow \mathbb{R}^{+}$qui ne dépend pas de $x$ et telle que la série $G(x, s)=$ 
$\sum_{\gamma \in \Gamma} e^{-s d_{\tilde{X}}(x, \gamma o)} h\left(d_{\tilde{X}}(x, \gamma o)\right)$ diverge si et seulement si $s \leq \delta(\Gamma)$. Lorsque $\Gamma$ est divergent, la fonction $h=1$ convient; sinon la construction de $h$ est plus délicate (cf. [Pa]). Une mesure de Patterson $\sigma_{x}$ est une valeur d'adhérence faible sur $\tilde{X} \cup \partial \tilde{X}$ lorsque $s \rightarrow \delta(\Gamma)^{+}$de la famille de mesures orbitales

$$
\sigma_{x, s}=\frac{1}{G(o, s)} \sum_{\gamma \in \Gamma} h(d(x, \gamma o)) e^{-s d_{\tilde{X}}(x, \gamma o)} \delta_{\gamma o} .
$$

Le support de ces mesures limites est toujours égal à $L(\Gamma)$.

Lorsque $\Gamma$ est divergent, la suite de mesures $\sigma_{x, s}$ converge lorsque $s \rightarrow \delta(\Gamma)^{+}$et la mesure $\sigma_{x}$ est portée par l'ensemble limite radial de $\Gamma$, c'est-à-dire l'ensemble des points $\xi$ de $L(\Gamma)$ tels qu'il existe une infinité de points de l'orbite $\Gamma o$ à distance bornée du rayon géodésique $[o, \xi[$.

Lorsque $\Gamma$ est convergent, la situation est très différente : en particulier, pour un groupe géométriquement fini convergent, les mesures $\sigma_{x}$ sont purement atomiques, portées par les orbites de points fixes paraboliques de $\Gamma$.

Mais dans tous les cas, les mesures $\sigma_{x}$ sont $\delta(\Gamma)$-conformes, c'est-à-dire qu'elles vérifient, pour $x, x^{\prime}$ dans $\tilde{X}$ :

$$
\frac{d \sigma_{x}}{d \sigma_{x^{\prime}}}(\xi)=e^{-\delta(\Gamma) \mathcal{B}_{\xi}\left(x, x^{\prime}\right)},
$$

où $\mathcal{B}_{\xi}\left(x, x^{\prime}\right)$ désigne la fonction de Busemann centrée en $\xi$. Les mesures $\sigma_{x}$ sont aussi $\Gamma$-invariantes : pour tout $\gamma \in \Gamma, \gamma^{*} \sigma_{x}=\sigma_{\gamma^{-1} x}$.

Dans la suite, nous fixerons une mesure de Patterson $\sigma_{x}$. Nous allons rappeler le procédé mis en lumière par D. Sullivan qui permet d'associer à cette famille une mesure sur $T^{1} X$ : c'est une mesure que nous noterons $\mu$, invariante sous l'action du flot géodésique et portée par son ensemble non-errant $\Lambda(\Gamma)$. Le fibré unitaire $T^{1} \tilde{X}$ est homéomorphe à $(\partial X \times \partial X-\Delta) \times \mathbb{R}$, où $\Delta$ désigne la diagonale : si $\tilde{v}$ est un vecteur de $T^{1} \tilde{X}$, de point base $x$, on lui associe le triplet $\left(\tilde{v}^{-}, \tilde{v}^{+},-t\right)$, où $\tilde{v}^{ \pm}$sont les extrémités positive et négative de la géodésique déterminée par $\tilde{v}$, et $t=\mathcal{B}_{\tilde{v}^{+}}(o, x)$. La mesure

$$
d \tilde{\mu}=e^{\delta(\Gamma)\left(\mathcal{B}_{\tilde{v}^{-}}(o, x)+\mathcal{B}_{\tilde{v}^{+}}(o, x)\right)} d \sigma\left(\tilde{v}^{-}\right) d \sigma\left(\tilde{v}^{+}\right) d t
$$

ne dépend pas du choix du point $x$, qu'elle est invariante sous l'action de $\Gamma$ et sous celle du flot géodésique sur $T^{1} \tilde{X}$; elle passe au quotient en une mesure $\mu$, appelée mesure de Patterson-Sullivan. Son support est l'ensemble non-errant $\Lambda(\Gamma)$; c'est une mesure de Radon, qui peut être finie ou infinie. Par exemple, si $\tilde{X}$ est un espace symétrique de rang 1 et $\Gamma$ est géométriquement fini, $\mu$ est toujours finie ([Su2], [CI]). Lorsque $\mu$ est finie, nous la supposerons toujours normalisée en une mesure de probabilité.

La mesure $\mu$ a deux propriétés essentielles. C'est d'une part sa structure de produit local par rapport au feuilletages stable/instable, d'autre part une propriété d'expansion uniforme .

Pour tout vecteur $\tilde{v} \in T^{1} \tilde{X}$, l'application $P_{\tilde{v}}$ de $W^{+}(\tilde{v})$ sur $\partial \tilde{X}-\left\{\tilde{v}^{-}\right\}$qui associe à un vecteur $w$ le bout positif $w^{+}$de la géodésique qu'il détermine est un homéomorphisme. On définit une mesure $\mu_{\tilde{v}}^{+}$sur $W^{+}(\tilde{v})$ en posant, pour tout borélien $A \subset T^{1} \tilde{X}$ :

$$
\mu_{\tilde{v}}^{+}(A)=\int \chi_{A}\left(P_{\tilde{v}}^{-1}(\xi)\right) e^{-\delta(\Gamma) \mathcal{B}_{\xi}(o, \tilde{\pi}(\tilde{v}))} d \sigma(\xi)
$$

Si $v \in T^{1} X$ est l'image de $\tilde{v}$ par la projection de revêtement, on note $\mu_{v}^{+}$l'image par cette projection de la mesure $\mu_{\tilde{v}}^{+}$restreinte à un domaine fondamental de l'action de $\Gamma$ sur $\tilde{X}$ : la mesure $\mu_{v}^{+}$est supportée sur la feuille $W^{+}(v)$. 
On définit de la même manière une famille de mesures $\left(\mu_{v}^{-}\right)_{v \in T^{1} X}$ sur les feuilles du feuilletage stable $W^{-}$.

Nous aurons besoin plus loin d'expliciter l'expression locale de la mesure $\mu_{\sigma}$. Soit $U$ un voisinage de $v$ sur sa feuille stable $W_{v}^{-}$; soit $\epsilon>0$. On remarque que tout vecteur $v \in T^{1} X$ est contenu dans un ouvert feuilleté $\mathcal{U}=\bigcup_{u \in U} \bigcup_{|s|<\epsilon} U_{\phi_{s} u}^{+}$où $U_{\phi_{s} u}^{+}$est un voisinage de $\phi_{s} u$ sur sa feuille fortement instable. La mesure $\mu$ restreinte à $\mathcal{U}$ a alors pour expression, si $A$ est un borélien contenu dans $\mathcal{U}$ :

$$
\mu(A)=\int_{U}\left(\int_{-\epsilon}^{\epsilon} \mu_{\phi^{s} u}^{+}\left(A \cap W_{\phi_{s} u}^{+}\right) e^{-\delta_{\Gamma} s} d s\right) d \mu_{v}^{-}(u)
$$

Comme les mesures $\sigma_{x}$ sont $\delta(\Gamma)$-conformes, on voit aussi que la famille $\left(\mu_{v}^{+}\right)_{v \in T^{1} X}$ possède la propriété d'expansion uniforme suivante. Pour tout $v \in T^{1} X$ et pour tout réel $t$, on a :

$$
\frac{d\left(\phi_{t}^{*} \mu_{\phi_{t} v}^{+}\right)}{d \mu_{v}^{+}}=e^{\delta(\Gamma) t}
$$

On utilisera dans la démonstration du Théorème 2 le fait que, pour tout borélien $A \subset T^{1}(X)$, l'application $v \rightarrow \mu_{v}^{+}(A)$ est borélienne : ceci découle de l'expression de $\mu_{v}^{+}$et de ce que l'application $\tilde{v} \rightarrow P_{\tilde{v}}^{-1}(\xi)$ est continue sur son ensemble de définition.

\section{§2. Partitions mesurables et Théorie de l'entropie.}

- Partitions mesurables.

On considère un espace mesuré $(M, \mathcal{M}, m)$ et on note $\overline{\mathcal{M}}$ la tribu obtenue en complétant la tribu $\mathcal{M}$ par les ensembles $m$-négligeables. Soit $\xi$ une partition de $M$; on appelle les éléments de $\xi$ des "atomes" et on note $\xi(x)$ l'atome contenant $x$. On note $M_{\xi}$ l'espace quotient de la partition, c'est-à-dire l'ensemble des atomes. On munit $M_{\xi}$ de la $\sigma$-algèbre $\mathcal{M}_{\xi}$ des éléments de $\overline{\mathcal{M}}$ qui sont réunion d'éléments de $\xi$. La mesure $m$ induit une mesure $\bar{m}$ sur $M_{\xi}$.

La partition $\xi$ est dite $m$-mesurable, s'il existe un ensemble de mesure pleine $N \subset M$, et une famille dénombrable $\left(A_{n}\right)_{n \in \mathbb{N}}$ d'éléments de $\mathcal{M}_{\xi}$ tels que pour tous les éléments distincts $\xi_{1}$ et $\xi_{2}$ de $\xi$, il existe $i \in \mathbb{N}$ tel que $\xi_{1} \cap N \subset A_{i}$ et $\xi_{2} \cap N \subset M-A_{i}$ (quitte à échanger $\xi_{1}$ et $\xi_{2}$ ). Une telle famille $\left(A_{n}\right)$ est appelée une base de la partition $\xi$. Si $\xi$ est une partition $m$-mesurable, l'espace $\left(M_{\xi}, \mathcal{M}_{\xi}, \bar{m}\right)$ est un espace de Lebesgue, c'est-à-dire qu'il est isomorphe à la réunion de l'intervalle $[0,1]$ muni de la mesure de Lebesgue et d'un ensemble discret de mesure finie.

On identifie deux partitions $m$-mesurables $\xi$ et $\xi^{\prime}$ lorsque, pour $m$-presque tout $x, \xi(x)=\xi^{\prime}(x)$.

Si $\xi$ et $\xi^{\prime}$ sont deux partitions $m$-mesurables, on dit que $\xi$ est plus fine que $\xi^{\prime}$ et l'on écrit $\xi \succ \xi^{\prime}$ si pour $m$-presque tout $x$, on a : $\xi(x) \subset \xi^{\prime}(x)$. Ceci définit un ordre partiel sur l'ensemble des (classes d'équivalence de) partitions mesurables.

Si $\xi$ et $\xi^{\prime}$ sont deux partitions $m$-mesurables, on note $\xi \vee \xi^{\prime}$ la partition $m$-mesurable obtenue en intersectant les éléments de $\xi$ et de $\xi^{\prime}$.

Si $\left(\xi_{n}\right)_{n \in \mathbb{N}}$ est une suite croissante de partitions mesurables, on note $\vee_{n \in \mathbb{N}} \xi_{n}$ la borne supérieure des partitions $\xi_{n}$, c'est-à-dire la plus petite partition mesurable qui majore toutes les $\xi_{n}$.

Une partition mesurable peut toujours être vue comme limite croissante d'une suite de partitions finies. Soit en effet $\xi_{n}$ la partition formée des intersections des ensembles $A_{i}$ et $M-A_{i}$ pour $i \leq n$. Alors $\xi_{n+1} \succ \xi_{n}$ et on a : $\vee_{n \in \mathbb{N}} \xi_{n}=\xi$.

Enfin, lorsque $\phi$ est une transformation de $(M, \mathcal{M}, m)$ dans lui-même, la partition $\phi^{-1} \xi=$ $\left\{\phi^{-1}(\xi(x)) \mid x \in M\right\}$ est une partition mesurable (on a bien sûr $\phi^{-1} \xi(x)=\phi^{-1}(\xi(\phi(x)))$. 
-Mesures conditionnelles.

Supposons que $m$ est une mesure de probabilité. À la donnée d'une partition $m$-mesurable $\xi$, on associe une famille de mesures conditionnelles sur les atomes de $\xi$. Précisément, pour presque tout $x \in M$, on peut définir une mesure de probabilité $m_{\xi(x)}$ sur l'atome $\xi(x)$; cette mesure a les propriétés suivantes :

(1) pour tout ensemble mesurable $A \subset M$, la fonction $x \rightarrow m_{\xi(x)}(A \cap \xi(x))$ est mesurable ;

(2) on a : $m(A)=\int_{M} m_{\xi(x)}(A \cap \xi(x)) d m(x)$.

Une famille de mesures $\left(m_{\xi(x)}\right)_{x}$ avec ces deux propriétés est appelée famille de probabilités conditionnelles pour $\xi$. Elle est définie de manière unique modulo $m$.

-Entropie.

Si $\xi$ est une partition mesurable finie de $(M, \mathcal{M}, m)$, on appelle entropie de $\xi$ relativement à $m$, le nombre

$$
H_{m}(\xi)=-\int_{M} \log m(\xi(x)) d m(x)=-\sum \log m\left(\xi_{i}\right) m\left(\xi_{i}\right),
$$

en convenant que $\infty .0=0$. L'entropie moyenne de $\xi$ est alors $h_{m}(\phi, \xi)=\inf _{n \geq 1} \frac{1}{n} H_{m}\left(\vee_{0}^{n-1} \phi^{-k} \xi\right)$. $L$ 'entropie de $\phi$ est la quantité $h_{m}(\phi)=\sup \left\{h_{m}(\phi, \xi)\right\}$, le suprémum étant pris sur toutes les partitions finies $\xi$.

On peut définir aussi l'entropie moyenne d'une partition mesurable décroissante (on dit qu'une partition mesurable $\xi$ est décroissante lorsque $\phi^{-1} \xi \succ \xi$ ). D'abord, si $\xi$ et $\xi^{\prime}$ sont deux partitions mesurables de $M$ telles que $\xi \succ \xi^{\prime}$, on pose

$$
H_{m}\left(\xi \mid \xi^{\prime}\right)=-\int \log m_{\xi^{\prime}(x)}(\xi(x)) d m(x)
$$

en convenant que $\int_{A} \infty \cdot d m(x)=0$ lorsque $m(A)=0$ et $=+\infty$ sinon. Si $\xi$ est une partition mesurable décroissante, on définit : $h_{m}(\phi, \xi)=H_{m}\left(\phi^{-1} \xi \mid \xi\right)$. Si $\xi$ est une partition finie, la partition $\xi^{+}=\vee_{n \in \mathbb{N}} \phi^{-n} \xi$ est une partition mesurable décroissante et on a : $h_{m}(\phi, \xi)=H_{m}\left(\phi^{-1} \xi^{+} \mid \xi^{+}\right)=h_{m}\left(\phi, \xi^{+}\right)$.

On dit que la partition $m$-mesurable $\xi$ est génératrice pour $\phi$ si la partition $\vee_{n \in \mathbb{N}} \phi^{-n} \xi$ est la partition en points.

Dans ce qui suit, nous appliquerons ces notions au cadre suivant : $M$ est l'ensemble non-errant $\Lambda(\Gamma)$ du flot géodésique sur $T^{1} X$, la tribu $\mathcal{M}$ est la tribu borélienne, et la transformation $\phi$ est l'un des temps $\phi_{\tau}$ du flot géodésique $\left(\phi_{t}\right)$.

On dit alors que la partition $m$-mesurable $\xi$ est subordonnée au feuilletage instable $W^{+}$, si pour $m$-presque tout $v \in M$, l'atome $\xi(v)$ est contenu dans la feuille $W^{+}(v)$ et est un voisinage de $v$ sur cette feuille.

Proposition 1. Soit $m$ une mesure de probabilité invariante par le flot géodésique sur $\Lambda(\Gamma)$ et soit $\phi=\phi_{\tau}$ un temps du flot géodésique. Supposons que $m$ est ergodique pour $\phi$. Alors il existe une partition m-mesurable $\xi$ de $\Lambda(\Gamma)$ qui est décroissante, génératrice pour $\phi$ et subordonnée au feuilletage $W^{+}$.

Démonstration. Ce résultat est classique dans le cas où $\phi$ est un difféomorphisme ergodique d'une variété compacte [LS] ; toutefois, nous en donnons la démonstration ici car certains points se simplifient du fait du caractère Anosov du flot $\left(\phi_{t}\right)$. 
Pour $u \in T^{1} X$ et $r>0$, on note $B^{+}(u, r)$ (resp. $\left.B^{-}(u, r)\right)$ la boule de centre $u$, et de rayon $r$ de la feuille $W^{+}(u)$ (resp. sur $W^{-}(u)$ ), pour la métrique riemannienne induite. Pour tout triplet $\left(r_{0}, r^{-}, r^{+}\right)$de réels strictement positifs, on définit la "cellule"

$$
\mathcal{C}\left(u, r_{0}, r_{-}, r_{+}\right)=\bigcup_{|s|<r_{0}} \phi_{s}\left(\cup_{v \in B^{-}\left(u, r_{-}\right)} B^{+}\left(v, r_{+}\right)\right) .
$$

C'est un voisinage ouvert de $u$ dans $T^{1} X$ et d'après les théorèmes de comparaison [CE], on a, pour tout $t>0$ :

$$
\mathcal{C}\left(\phi_{t} u, r_{0}, e^{-\beta t} r_{-}, e^{\alpha t} r_{+}\right) \subset \phi_{t}\left(\mathcal{C}\left(u, r_{0}, r_{-}, r_{+}\right)\right) \subset \mathcal{C}\left(\phi_{t} u, r_{0}, e^{-\alpha t} r_{-}, e^{\beta t} r_{+}\right)
$$

Fixons un vecteur $u \in \Lambda(\Gamma)$ et posons pour simplifier $\mathcal{C}(u, r)=\mathcal{C}(u, r, r, r)$. On munit la cellulle $\mathcal{C}(u, r)$ de "coordonnées" de la façon suivante : un point $x \in \mathcal{C}(u, r)$ est représenté par $s \in]-r, r[$ tel que $x=\phi_{r}(w)$ où $w \in \cup_{v \in B^{-}(u, r)} B^{+}(v, r) ; w$ est représenté par $v \in B^{-}(u, r)$ tel que $w \in$ $B^{+}(v, r)$. Choisissons un réel $0<\rho \leq i n j(\pi(u) / 4$. On définit alors sur $\mathcal{C}(u, \rho)$ une fonction $h: x \rightarrow \sup \left(|s|, d^{-}(w, v), d^{+}(u, v)\right):$ par construction, la ligne de niveau $h^{-1}\{r\}$, pour $\left.r \in\right] 0, \rho[$, est égale à la frontière de la cellule $\mathcal{C}(u, r)$. D'autre part, la régularité des feuilletages stable et instable entraînent que la fonction $h$ est Hölder sur $\mathcal{C}(u, \rho)$, celle-ci étant munie de la distance induite par la distance riemannienne de $T^{1} X$.

Pour $r>0$, considérons la partition $\hat{\xi}_{r}$ de $T^{1} X$ dont les atomes sont d'une part les intersections $W^{+}(v) \cap \mathcal{C}(u, r)$ et aussi $T^{1} X-\mathcal{C}(u, r)$. Nous fixerons la valeur de $r$ plus tard, mais supposons pour le moment $r \leq i n j(\pi(u)) / 4$ où $i n j(\pi(u))$ est le rayon d'injectivité en la projection $\pi(u)$ du vecteur $u$ sur $X$. Cette propriété sera utilisée, dans le Lemme 2; elle garantit que si $v$ et $w$ sont deux points de $T^{1} X$ tels que $W^{+}(v)=W^{+}(w)$ mais $\hat{\xi}_{r}(v) \neq \hat{\xi}_{r}(w)$, alors $d^{+}(v, w) \geq \frac{r}{2}$, où $d^{+}$est la distance induite par la métrique riemannienne sur les feuilles de $W^{+}$.

On définit $\xi_{r}=\vee_{0}^{\infty} \phi^{n} \hat{\xi}_{r}: \xi_{r}$ est une partition décroissante de $T^{1} X$ et en particulier induit une partition décroissante de $\Lambda(\Gamma)$, toujours notée $\xi_{r}$. Nous allons montrer que l'on peut choisir $r$ de sorte que $\xi_{r}$ vérifie les conclusions de la Proposition 1.

Montrons d'abord que $\xi_{r}$ est $m$-mesurable. Il est clair que la partition $\hat{\xi}_{r}$ l'est, puisque l'espace des atomes de $\hat{\xi}_{r}$ contenus dans $\mathcal{C}(u, r)$ s'identifie à $\left.B^{-}(u, r) \times\right]-r, r[$; il suffit alors de choisir pour base la famille dénombrable $\left(A_{i}\right)_{i}$ formée du complémentaire de $\mathcal{C}(u, r)$ et des saturés par $\hat{\xi}_{r}$ des ouverts d'une base dénombrable de $\left.B^{-}(u, r) \times\right]-r, r\left[\right.$. On en déduit que $\xi_{r}$ est mesurable, la base correspondante étant formée des $\phi^{k}\left(A_{i}\right)$, pour $k \in \mathbb{N}$.

Vérifions que pour tout $r>0$ et pour $m$-presque tout $v$, l'atome $\xi_{r}(v)$ est contenu dans $W^{+}(v)$. Puisque $u \in \Lambda(\Gamma)$, la mesure $m(\mathcal{C}(u, r))$ est strictement positive. L'ergodicité de $m$ assure donc en particulier que pour $m$-presque tout $v$, il existe un entier $n>0$ tel que $\phi^{-n} v \in \mathcal{C}(u, r)$; ainsi $\xi_{r}(v)$ est inclus dans $\phi^{n}\left(\hat{\xi}_{r}\left(\phi^{-n} v\right)\right)$, et a fortiori dans $W^{+}(v)$. Un argument analogue donne que $\xi_{r}$ est génératrice pour $\phi$. En effet, pour $m$-presque tout $v$, il existe une suite $\left(n_{k}\right)$ tendant vers $+\infty$ telle que $\phi^{n_{k}} v \in \mathcal{C}(u, r)$ : comme le diamètre des atomes de la partition $\hat{\xi}_{r}$ contenus dans $\mathcal{C}(u, r)$ est majoré, le diamètre de l'atome $\phi^{-n_{k}} \hat{\xi}_{r}\left(\phi^{n_{k}} v\right)$ tend vers 0 quand $k \rightarrow \infty$.

Remarquons que l'argument d'ergodicité précédent montre aussi que pour $m$-presque tout $v$, l'atome $\xi_{r}(v)$ est contenu dans un ensemble $\phi^{k}\left(A_{i}\right)$ qui est relativement compact. En particulier, lorsqu'on décrit $\xi_{r}$ comme limite croissante d'une suite $\left(\xi_{n}\right)$ de partitions finies, alors pour $m$ presque tout $v$, l'atome $\xi_{r}(v)$ est contenu dans un atome $\xi_{n}(v)$ qui est relativement compact. Cette remarque sera utilisée dans le Fait 9 , au cours de la démonstration du Théorème 2.

Il est plus délicat de montrer que l'on peut choisir $r>0$ de sorte que pour $m$-presque tout $v$, l'atome $\xi_{r}(v)$ soit un voisinage de $v$ sur la feuille $W^{+}(v)$. Pour cela, rappelons que d'après le 
caractère Anosov du flot $\left(\phi_{t}\right)$ sur $T^{1} X$, il existe un réel $A>0$ tel que si un vecteur $w \in W^{+}(v)$ vérifie $d^{+}(v, w)<i n j_{X}(\pi(v))$, alors pour tout temps $t \geq 0$, on aura $d^{+}\left(\phi^{-t} v, \phi^{-t} w\right) \leq A e^{-\alpha t} d^{+}(v, w)$. Posons alors

$$
\beta(v)=\inf _{n \geq 0}\left\{\frac{e^{n \alpha}}{2 A} d^{+}\left(\phi^{-n} v, \partial \mathcal{C}(u, r)\right), \frac{r}{2 A}, \operatorname{inj}(\pi(v))\right\} .
$$

\section{Lemme 2.}

(1) Si $w \in W^{+}(v)$ vérifie $d^{+}(v, w)<\beta(v)$, alors pour tout entier $n \geq 0$, on $a: \hat{\xi}_{r}\left(\phi^{-n} v\right)=$ $\hat{\xi}_{r}\left(\phi^{-n} w\right)$.

(2) On peut choisir $0<r<\operatorname{inj}(\pi(u) / 4$ de sorte que pour $m$-presque tout $v$ on ait $\beta(v)>0$.

Admettons ce résultat pour le moment et terminons la démonstration de la Proposition 1. Choisissons la constante $r$ de sorte que (2) soit vérifié. Alors, d'après (1), on trouve que pour un ensemble de mesure pleine de $v \in \Lambda(\Gamma)$, la boule ouverte $B^{+}(v, \beta(v))$ de $W^{+}(v)$ est contenue dans $\xi_{r}(v)$. La partition $\xi_{r}$ est donc subordonnée à $W^{+}$.

Démonstration $d u$ Lemme 2. Soit $w \in W^{+}(v)$ tel que $d^{+}(v, w)<\beta(v)$. Alors, par définition de $\beta(v)$, on a $d^{+}\left(\phi^{-n} v, \phi^{-n} w\right) \leq A e^{-\alpha n} d^{+}(v, w)$, d'où $d^{+}\left(\phi^{-n} v, \phi^{-n} w\right) \leq \frac{1}{2} d^{+}\left(\phi^{-n} v, \partial \mathcal{C}\right)$ et aussi $d^{+}\left(\phi^{-n} v, \phi^{-n} w\right) \leq \frac{r}{2}$. La première inégalité montre alors qu'on ne peut avoir simultanément $\phi^{-n} v \in \mathcal{C}(u, r)$ et $\phi^{-n} w \in \mathcal{C}(u, r)^{c}$ (ou vice versa) ; la seconde que si $\phi^{-n} v$ et $\phi^{-n} w$ appartiennent à $\mathcal{C}(u, r)$ alors $\hat{\xi}\left(\phi^{-n} v\right)=\hat{\xi}\left(\phi^{-n} w\right)$.

Pour montrer (2), on utilise un argument de théorie de la mesure (cf. [LS], Proposition 3.2).

Fait 3. Soit $\nu$ une mesure de probabilité supportée sur un intervalle $] 0, \rho\left[\subset \mathbb{R}^{+}\right.$et a $\left.\in\right] 0,1[$. Alors la mesure de Lebesgue de l'ensemble $\{r \in] 0, \rho\left[\quad \mid \quad \sum_{0}^{\infty} \nu\left[r-a^{k}, r+a^{k}\right]<\infty\right\}$ est égale à $\rho$.

Prolongeons la fonction $h$ en une fonction Hölder en la définissant égale à $\rho$ dans le complémentaire de la couronne $\mathcal{C}(u, \rho)$. Appliquons le Fait 3 à la mesure image de la mesure $m$ par l'application $h$. L'invariance de $m$ par $\phi$ entraîne alors que l'ensemble des $r \in] 0, \rho$ [ tels que $\sum_{k=0}^{\infty} m\left\{v \in T^{1} X:\left|h\left(\phi^{-k} v\right)-r\right| \leq a^{k}\right\}<\infty$ est de mesure de Lebesgue pleine.

Or, puisque $h$ est Hölder, il existe des constantes $A^{\prime}>0$, et $\left.\kappa \in\right] 0,1[$ telles que si $d(w, \partial \mathcal{C}(u, r)) \leq$ $\tau$, alors on a : $|h(w)-r| \leq\left(A^{\prime} \tau\right)^{\kappa}$. Soit $K$ l'ensemble des $\left.r \in\right] 0, \rho[$ tels que

$$
\sum_{k=0}^{\infty} m\left\{v \in T^{1} X \mid d\left(\phi^{-k} v, \partial \mathcal{C}(u, r)\right) \leq \frac{e^{-k \alpha}}{A^{\prime}}\right\}<\infty
$$

cet ensemble sera a fortiori de mesure de Lebesgue pleine. Si on choisit $r \in K$ tel que $m\left(\bigcup_{k} \phi^{k} \partial \mathcal{C}(u, r)\right)=0$, on obtient le résultat escompté.

Le résultat suivant montre l'utilité de la partition $\xi$ construite dans la Proposition 1 puisqu'elle va nous permettre de calculer l'entropie de la mesure $m$.

Proposition 4. Soient $m$ une mesure $\phi$-invariante ergodique et $\xi$ la partition mesurable construite dans la Proposition 1. Alors $h_{m}(\phi)=h_{m}(\phi, \xi)$.

$\mathrm{Au}$ cours de la démonstration, nous utiliserons des partitions finies d'un type particulier que nous allons maintenant introduire. Rappelons que le feuilletage faiblement instable de $T^{1} X$ a pour feuilles les orbites par le flot géodésique $\left(\phi_{t}\right)$ des feuilles instables, c'est-à-dire les réunions $W_{f}^{+}(v)=\bigcup_{t} \phi_{t} W^{+}(v)$. Sous les hypothèses de la Proposition 4, on a : 
Fait 5. Il existe une partition finie $\hat{\mathcal{P}}$ de $\Lambda(\Gamma)$ telle que la partition $\mathcal{P}=\vee_{0}^{\infty} \phi^{n} \hat{\mathcal{P}}$ possède les propriétés suivantes :

(1) pour $m$-presque tout $v \in T^{1} X$, l'atome $\mathcal{P}(v)$ est contenu dans la feuille faiblement instable $W_{f}^{+}(v)$.

(2) pour presque tout $v$ et pour tout $w \in \mathcal{P}(v)$, on $a: W^{+}(w) \cap \mathcal{P}(v)=\xi(w) \cap \mathcal{P}(v)$.

De plus, on peut choisir la partition finie $\hat{\mathcal{P}}$ de sorte que $h_{m}(\phi, \mathcal{P})$ soit arbitrairement proche de $h_{m}(\phi)$.

Démonstration. Considérons la cellule $\mathcal{C}(u, r)$ utilisée dans la construction de $\xi$. On définit maintenant $\hat{\mathcal{P}}$ comme la partition en deux atomes : $\mathcal{C}(u, r)$ et son complémentaire $T^{1} X-\mathcal{C}(u, r)$. La partition $\mathcal{P}=\vee_{0}^{\infty} \phi^{n} \hat{\mathcal{P}}$ est mesurable. Par ergodicité de la mesure $m$, pour $m$-presque tout $v \in T^{1} X$, il existe une suite $\left(n_{k}\right)$ tendant vers $+\infty$ et telle que $\phi^{-n_{k}} v \in \mathcal{C}(u, r)$. Observons qu'il existe $r^{\prime}$ tel que pour tout $u^{\prime} \in \mathcal{C}(u, r)$, on a : $\mathcal{C}(u, r) \subset \mathcal{C}\left(u^{\prime}, r^{\prime}\right)$. Puisque $\mathcal{C}(u, r) \subset \mathcal{C}\left(\phi^{-n_{k}} v, r^{\prime}\right)$, on aura alors $\mathcal{P}(v) \subset \mathcal{C}\left(v, r^{\prime}, e^{-\alpha n_{k}} r^{\prime}, e^{\beta n_{k}} r^{\prime}\right)$ pour tout $k$. Comme $\cap_{k} \mathcal{C}\left(v, r^{\prime}, e^{-\alpha n_{k}} r^{\prime}, e^{\beta n_{k}} r^{\prime}\right) \subset W_{f}^{+}(v)$, on a bien : $\mathcal{P}(v) \subset W_{f}^{+}(v)$ pour $m$-presque tout $v$.

D'après la construction de $\xi$, pour presque tout $v$ et pour tout $w \in \mathcal{P}(v)$, on a : $\xi(w)=$ $\xi(w) \cap \mathcal{P}(v)=W^{+}(w) \cap \mathcal{P}(v)$.

Soit maintenant $\mathcal{A}$ une partition finie d'entropie proche de $h_{m}(\phi)$. Alors, la partition $\hat{\mathcal{P}}^{\prime}=\hat{\mathcal{P}} \vee \mathcal{A}$ est finie, son entropie est proche de $h_{m}(\phi)$ et $\mathcal{P}^{\prime}=\vee_{0}^{\infty} \phi^{n} \hat{\mathcal{P}}^{\prime}$ vérifie (1) et (2).

Démonstration de la Proposition 4. Soit $\xi$ la partition mesurable construite dans la Proposition 1. Il nous faut montrer d'une part que $h_{m}(\phi, \xi)$ est finie et d'autre part que pour toute partition $\mathcal{P}$ d'entropie finie, on a $: h_{m}(\phi, \xi) \geq h_{m}(\phi, \mathcal{P})$. Il suffit de considérer les partitions $\mathcal{P}$ construites dans le Fait 5 . Soit $\mathcal{P}$ une telle partition ; on définit une nouvelle partition mesurable en posant $\eta=\xi \vee \mathcal{P}$.

Remarquons d'abord que $h_{m}(\phi, \eta)=h_{m}(\phi, \xi)$. Puisque $\eta$ est plus fine que $\xi$, il suffit de vérifier que $h_{m}(\phi, \eta) \leq h_{m}(\phi, \xi)=H_{m}(\xi \mid \phi \xi)$. Or on a :

$$
\begin{gathered}
h_{m}(\phi, \eta)=H_{m}(\xi \vee \mathcal{P} \mid \phi \xi \vee \phi \mathcal{P})=H_{m}(\xi \mid \phi \xi \vee \phi \mathcal{P})+H_{m}(\mathcal{P} \mid \xi \vee \phi \mathcal{P}) \\
=H_{m}(\xi \mid \phi \xi \vee \phi \mathcal{P})+H_{m}\left(\phi \mathcal{P} \mid \phi \xi \vee \phi^{2} \mathcal{P}\right)=H_{m}\left(\xi \vee \phi \mathcal{P} \mid \phi \xi \vee \phi^{2} \mathcal{P}\right) .
\end{gathered}
$$

De même on a, pour tout $n \geq 0: h_{m}(\phi, \eta)=H_{m}\left(\xi \vee \phi^{n} \mathcal{P} \mid \phi \xi \vee \phi^{n+1} \mathcal{P}\right)$. Or,

$$
H_{m}\left(\xi \vee \phi^{n} \mathcal{P} \mid \phi \xi \vee \phi^{n+1} \mathcal{P}\right) \leq H_{m}(\xi \mid \phi \xi)+H_{m}\left(\mathcal{P} \mid \phi^{-n+1} \xi \vee \phi \mathcal{P}\right)
$$

Comme le dernier terme tend vers 0 quand $n \rightarrow \infty$, on a que $h_{m}(\phi, \eta) \leq h_{m}(\phi, \xi)$.

Avant de commencer la démonstration de la Proposition 4, nous allons décrire un peu plus les atomes de $\mathcal{P}$. La partition $\eta$ induit une partition de $\mathcal{P}(v)$, notée $\eta \mid \mathcal{P}(v)$ dont les atomes sont les $\eta(w)$ pour $w \in \mathcal{P}(v)$. Pour $m$-presque tout $v$, cette partition est aussi la partition en feuilles instables d'après le Fait $5,(2)$. Si $w$ et $w^{\prime}$ sont deux points de $\mathcal{P}(v)$, on pose $d_{T}\left(\eta(w), \eta\left(w^{\prime}\right)\right)=$ $\left|\mathcal{B}_{v^{-}}\left(\pi w, \pi w^{\prime}\right)\right|$ où $\mathcal{B}$ désigne la fonction de Busemann. On vérifie que $d_{T}$ est une distance sur $\eta \mid \mathcal{P}(v)$, que nous appellerons "distance transverse". En d'autres termes, on a identifié l'espace des atomes de la partition $\eta \mid \mathcal{P}(v)$ à un intervalle $I_{v}$ de $\mathbb{R}$ en associant à $\eta(w)$ le point d'intersection $W^{+}(w) \cap\left(\phi_{t} v\right)_{t \in \mathbb{R}}$; dans cette identification, la distance $d_{T}$ correspond à la distance euclidienne sur $\mathbb{R}$. De plus, $\phi$ induit une isométrie de $\left(\eta \mid \mathcal{P}(v), d_{T}\right)$ sur $\left(\eta \mid \mathcal{P}(\phi v), d_{T}\right)$. 
Montrons maintenant que l'entropie $h_{m}(\phi, \xi)=h_{m}(\phi, \eta)=H_{m}\left(\phi^{-1} \eta \mid \eta\right)$ est finie. Par définition,

$$
H_{m}\left(\phi^{-1} \eta \mid \eta\right)=-\int \log m_{\eta(v)}\left(\phi^{-1} \eta(v)\right) d m(v),
$$

qui, d'après le choix de la partition $\xi$, vaut aussi :

$$
-\int \log m_{\eta(v)}\left(\phi^{-1} \mathcal{P}(v)\right) d m(v)
$$

On définit une fonction mesurable sur $M$ en posant $g(v)=m_{\eta(v)}\left(\phi^{-1} \mathcal{P}(v)\right)$; on veut donc montrer que $-\log g$ est intégrable.

Fixons un atome $\mathcal{P}(v)$. Pour presque tout $v$, la fonction $g$ induit une fonction sur l'espace des atomes de $\eta \mid \mathcal{P}(v)$, c'est-à-dire sur l'intervalle $I_{v}$; cet intervalle porte la mesure image de la mesure $m_{\mathcal{P}(v)}$, que nous noterons toujours $m_{\mathcal{P}(v)}$. On définit $g_{\delta}$ comme la moyenne de $g$ relativement à $m_{\mathcal{P}(v)}$ sur l'intervalle $I(w, \delta) \subset I_{v}$ de centre $\eta(w)$ et de rayon $\delta$ pour la distance transverse, c'est-à-dire :

$$
g_{\delta}(w)=\frac{1}{m_{\mathcal{P}(v)}(I(w, \delta))} \int_{I(w, \delta)} g d m_{\mathcal{P}(v)} .
$$

On introduit la fonction "maximale" $g_{*}(w)=\inf _{\delta} g_{\delta}(w)$.

Le Théorème de dérivation de Lebesgue donne $\lim _{\delta \rightarrow 0} g_{\delta}(w)=g(w)$, pour presque tout $w \in I_{v}$; appliqué à chaque atome, on a donc $g_{\delta}(v) \rightarrow g(v)$, pour presque tout $v$.

D'autre part, la partition $\mathcal{P}$ est d'entropie finie; donc pour presque tout $v$, la partition $\phi^{-1} \mathcal{P} \mid \mathcal{P}(v)$ est d'entropie finie. En raisonnant, comme dans [LS, p. 525] on trouve que $-\log g_{*}$ est intégrable et que :

$$
-\int_{\mathcal{P}(v)} \log g_{*} d m_{\mathcal{P}(v)} \leq H_{m_{\mathcal{P}(v)}}\left(\phi^{-1} \mathcal{P}\right)+\log 2+1 .
$$

En intégrant par rapport à $m$, il vient, puisque $g \geq g_{*}$ :

$$
-\int \log g d m \leq-\int \log g_{*} d m \leq H_{m}\left(\phi^{-1} \mathcal{P} \mid \mathcal{P}\right)+\log 2+1 .
$$

L'entropie de $\xi$ est donc finie.

Montrons enfin que $h_{m}(\phi, \eta) \geq h_{m}(\phi, \mathcal{P})$, ce qui démontrera la Proposition 4, puisque $h_{m}(\phi, \mathcal{P})$ peut être choisie arbitrairement proche de $h_{m}(\phi)$. Nous utiliserons le résultat suivant.

Lemme 6. Soit $c \in] 0,1[$. Pour $m$-presque tout $v$, on $a$ :

$$
\liminf _{n \rightarrow \infty}-\frac{\log m_{\mathcal{P}(v)}\left(I\left(v, e^{-c n}\right)\right)}{n} \geq(1-c)\left(h_{m}(\phi, \mathcal{P})-h_{m}(\phi, \eta)-c\right) .
$$

Démonstration. Nous allons adapter l'argument de [LY, Proposition 5.1]. Notons $p$ la partie entière de $n(1-c)$; fixons $\delta>0$. On a :

$$
m_{\mathcal{P}(v)}(I(v, \delta)) \leq \Pi_{0}^{p-1} \frac{m_{\mathcal{P}\left(\phi^{k} v\right)}\left(I\left(\phi^{k} v, \delta\right)\right)}{m_{\mathcal{P}\left(\phi^{k+1} v\right)}\left(I\left(\phi^{k+1} v, \delta\right)\right)}
$$


La mesure $m$ étant $\phi$-invariante et la partition $\mathcal{P}$ décroissante, on a, par la propriété de transitivité des mesures conditionnelles que, pour $m$-presque tout $w$ et pour tout ensemble mesurable $A \subset$ $\mathcal{P}(\phi w)$ :

$$
m_{\mathcal{P}(\phi w)}(A)=\frac{m_{\mathcal{P}(w)}\left(\phi^{-1} A\right)}{m_{\mathcal{P}(w)}\left(\phi^{-1} \mathcal{P}(w)\right)}
$$

Appliquons cette égalité aux points $\phi^{k} v$ et aux boréliens $I\left(\phi^{k} v, \delta\right)$ pour $k=0,1, \cdots, p-1$; on obtient alors

$$
\begin{gathered}
\frac{1}{n} \log m_{\mathcal{P}(v)}(I(v, \delta)) \leq I_{n}(v, \delta)+J_{n}(v), \quad \text { où } \\
I_{n}(v, \delta)=-\frac{1}{n} \sum_{k=0}^{p-1} \log \frac{m_{\mathcal{P}\left(\phi^{k} v\right)}\left(\phi^{-1} I\left(\phi^{k+1} v, \delta\right)\right)}{m_{\left.\mathcal{P}\left(\phi^{k} v\right)\right)}\left(I\left(\phi^{k} v, \delta\right)\right)} \text { et } \\
J_{n}(v)=\frac{1}{n} \sum_{k=0}^{p-1} \log m_{\mathcal{P}\left(\phi^{k} v\right)}\left(\left(\phi^{-1} \mathcal{P}\right)\left(\phi^{k} v\right)\right) .
\end{gathered}
$$

D'après le Théorème ergodique, pour $m$-presque tout $v$, on a, lorsque $n \rightarrow \infty$ :

$$
J_{n}(v) \rightarrow-(1-c) H_{m}\left(\phi^{-1} \mathcal{P} \mid \mathcal{P}\right)=-(1-c) h_{m}(\phi, \mathcal{P})
$$

Par ailleurs, $\phi^{-1} I\left(\phi^{k+1} v, \delta\right)=I\left(\phi^{k} v, \delta\right) \cap \phi^{-1} \mathcal{P}\left(\phi^{k} v\right) ;$ donc,

$$
I_{n}(v, \delta)=-\frac{1}{n} \sum_{k=0}^{p-1} \log g_{\delta}\left(\phi^{k}(v)\right)
$$

Comme pour presque tout $v, g_{\delta}(v) \rightarrow g(v)$, il existe une fonction mesurable $v \rightarrow \delta(v)$ telle que $-\log g_{\delta}(v) \leq-\log g(v)+c / 2$, dès que $\delta \leq \delta(v)$. On a vu plus haut que $-\int \log g_{*} d m<\infty$; donc pour une certaine constante $\delta_{c}>0$ on a $\int_{\left\{\delta(v) \leq \delta_{c}\right\}}-\log g_{*}(v) d m(v) \leq c / 2$. Posons : $A_{c}=\{v \in$ $\left.M \mid \delta(v)>\delta_{c}\right\}$. Pour tout entier $n$ vérifiant $e^{-c n} \leq \delta_{c}$, on a :

$$
I_{n}\left(v, e^{-c n}\right)=\frac{1}{n} \sum_{\phi^{k} v \in A_{c}}+\frac{1}{n} \sum_{\phi^{k} v \notin A_{c}} \leq \frac{1}{n} \sum_{\phi^{k} v \in A_{c}}\left(-\log g\left(\phi^{k} v\right)+\frac{c}{2}\right)-\frac{1}{n} \sum_{\phi^{k} v \notin A_{c}} \log g_{*}\left(\phi^{k} v\right) .
$$

D'où, d'après le théorème ergodique, presque tout $v$ vérifie :

$\limsup I_{n}\left(v, e^{-c n}\right) \leq(1-c)\left(\int-\log g d m+\frac{c}{2}+\int_{M-A_{c}}-\log g_{*} d m\right) \leq(1-c)\left(\int_{M}-\log g d m+c\right)$.

En utilisant que $\int_{M}-\log g d m=h_{m}(\phi, \eta)$, on obtient le Lemme 6 .

Pour terminer la démonstration de la Proposition 4, on remarque que le terme de gauche dans l'inégalité du Lemme 6 est majoré par $c$. En effet, pour toute mesure de Radon $\nu$ sur un intervalle $I$ de $\mathbb{R}$, on a, pour $\nu$-presque tout $x$ :

$$
\limsup _{\epsilon \rightarrow 0} \frac{\log \nu(I(x, \epsilon))}{\log \epsilon} \leq 1 .
$$


Appliqué à la mesure $m_{\mathcal{P}(v)}$ sur l'intervalle $\eta \mid \mathcal{P}(v)$, on trouve que pour $m$-presque tout point de $M$,

$$
\liminf _{n \rightarrow \infty}-\frac{\log m_{\mathcal{P}(v)}\left(I\left(v, e^{-c n}\right)\right)}{n} \leq c .
$$

En faisant tendre $c$ vers 0 dans l'inégalité obtenue au Lemme 6 , on obtient alors : $h_{m}(\phi, \eta) \geq$ $h_{m}(\phi, \mathcal{P})$.

Pour terminer cette section, supposons maintenant que la mesure de Patterson-Sullivan $\mu$ est finie et soit $\xi$ une partition $\mu$-mesurable subordonnée au feuilletage $W^{+}$. Nous allons maintenant interprêter les mesures conditionnelles $\mu_{\xi(v)}$ en termes des mesures $\mu_{v}^{+}$(cf. $\S 1$ ). On peut recouvrir un ensemble de mesure pleine de $T^{1} X$ par une famille d'ouverts feuilletés $\mathcal{U}_{i}$ comme ceux de $\S 1$. On définit alors une partition $\iota$ de $T^{1} X$ dont les atomes sont les ouverts $U_{w}$ pour $w \in U_{i}$ et le complémentaire de $\cup \mathcal{U}_{i}$ : cette partition est mesurable.

Considérons la partition $\xi^{\prime}=\xi \vee \iota$ qui raffine $\xi$ et $\iota$. D'après la structure de produit local de la mesure $\mu$, et puisque pour $\mu$-presque tout $v$, l'atome $\xi^{\prime}(v)$ est un voisinage relativement compact de $v$ sur $W_{v}^{+}$, et a donc une $\mu_{v}^{+}$-mesure positive, on a :

$$
\mu_{\xi^{\prime}(v)}=\frac{1}{\mu_{v}^{+}\left(\xi^{\prime}(v)\right)} \mu_{v}^{+}
$$

La propriété de transitivité des mesures conditionnelles [Ro] dit aussi que pour $\mu$-presque tout $v$, on a :

$$
\mu_{\xi^{\prime}(v)}=\frac{1}{\mu_{\xi(v)}\left(\xi^{\prime}(v)\right)} \mu_{\xi(v)} .
$$

On en déduit que pour $\mu$-presque tout $v$, on a :

$$
\mu_{\xi(v)}=\frac{1}{\mu_{v}^{+}(\xi(v))} \mu_{v}^{+} .
$$

\section{§3. Démonstration du Théorème 2.}

Les outils sont maintenant en place pour démontrer le Théorème 2. Rappelons que l'entropie $h_{m}\left(\left(\phi_{t}\right)\right)$ d'une mesure $m$ pour un flot $\left(\phi_{t}\right)$ est par définition celle de $\phi_{1}$ le temps 1 du flot ; par homogénéité, c'est aussi, pour $\tau>0, h_{m}\left(\left(\phi_{t}\right)\right)=\frac{1}{\tau} h_{m}\left(\phi_{\tau}\right)$. Pour calculer l'entropie $h_{m}\left(\phi_{\tau}\right)$, nous utiliserons une partition $m$-mesurable fournie par la Proposition 1. Pour construire ces partitions, une hypothèse était que la mesure $m$ soit ergodique pour $\phi_{\tau}$ : nous utiliserons le lemme bien connu suivant (cf. [PS], Theorem 1, pour un énoncé plus général). Par souci de clarté, nous en proposons une démonstration.

Lemme 7. Soit $(M, m)$ un espace de probabilité tel que $L^{2}(M, m)$ soit séparable. Soit $\left(T_{t}\right)$ un groupe à 1 paramètre de transformations de $M$ préservant la mesure. Si $m$ est ergodique pour le flot $\left(\phi_{t}\right)$, alors pour tous les temps $\tau \in \mathbb{R}$, sauf ceux d'un ensemble au plus dénombrable, $m$ est ergodique pour la transformation $\phi_{\tau}$.

Démonstration. On note $\left(U_{t}\right)$ le groupe de transformations unitaires de $L^{2}(M, m)$ induit par l'action de $\left(T_{t}\right)$ sur $M$. Soit $H$ le sous-espace de $L^{2}(M, m)$ formé des fonctions d'intégrale nulle. Soit $\tau$ un temps tel que $\phi_{\tau}$ n'est pas ergodique. Alors $U_{\tau}$ fixe un vecteur non-nul $v \in H$. Soit $K$ le sous-espace fermé de $H$ engendré par l'orbite $\left(U_{t} v\right)$. La représentation de $\mathbb{R}$ restreinte à $K$ se factorise par une représentation du cercle $\mathbb{R} / \tau \mathbb{Z}$. L'espace $K$ est donc somme orthogonale, indexée par 
$\mathbb{Z}$, de sous-espaces $K_{n}$, correspondants aux caractères $t \rightarrow e^{2 i \pi n t / \tau}$ du groupe $\mathbb{R} / \tau \mathbb{Z}$. L'ergodicité $\mathrm{du}$ flot $\left(T_{t}\right)$ signifie que la représentation de $\mathbb{R}$ n'a pas de vecteur non-nul invariant; donc $K_{0}=\{0\}$. Soit $K_{n} \neq\{0\}$, l'un de ces sous-espaces, et $y \neq 0$ un vecteur de $K_{n}$ : on a $U_{t} y=e^{2 i \pi n t / \tau} y$. Or, si $y, y^{\prime}$ sont deux vecteurs de $H$ associés à des caractères différents $t \rightarrow e^{2 i \pi n t / \tau}$ et $t \rightarrow e^{2 i \pi n^{\prime} t / \tau^{\prime}}$ (c'est-à-dire tels que $n / \tau \neq n^{\prime} / \tau^{\prime}$ ) alors $y$ et $y^{\prime}$ sont orthogonaux. Comme $H$ est séparable, on en déduit le lemme 7.

Nous pourrons donc toujours choisir, une mesure de probabilité ergodique $m$ étant fixée, un temps $\tau>0$ tel que $m$ soit ergodique pour $\phi_{\tau}$.

Nous allons d'abord montrer que lorsque la mesure de Patterson-Sullivan $\mu$ est finie, son entropie est égale à $\delta(\Gamma)$. D'après le Théorème de Hopf, si $\mu$ est finie, alors elle est ergodique pour le flot $\left(\phi_{t}\right)$. Soit $\phi=\phi_{\tau}$ un temps tel que $\mu$ est ergodique pour $\phi_{\tau}$ et choisissons une partition $\mu$-mesurable vérifiant les conditions de la Proposition 1.

Comme on l'a remarqué dans la section $\S 2$, on a :

$$
\mu_{\xi(v)}=\frac{1}{\mu_{v}^{+}(\xi(v))} \mu_{v}^{+}
$$

La propriété d'expansion uniforme de $\mu_{v}^{+}$sous l'action de $\phi_{\tau}$ se traduit alors par:

$$
-\log \mu_{\xi(v)}\left(\phi^{-1} \xi(v)\right)=\delta(\Gamma) \tau+F(v)-F(\phi v)
$$

en posant $F(v)=\log \mu_{v}^{+}(\xi(v))$. La fonction $F$ est $\mu$-mesurable puisque la partition $\xi$ est $\mu^{-}$ mesurable ; elle est finie sur une ensemble de $\mu$-mesure pleine puisque pour $\mu$-presque tout $v$, l'atome $\xi(v)$ est un voisinage de $v$ sur $W^{+}(v)$. On voit aussi sur la formule que le cobord $v \rightarrow F(\phi v)-F(v)$ est majoré par $\delta(\Gamma) \tau$. On utilise alors le Lemme classique suivant. Ne connaissant pas de référence précise, nous en proposons une démonstration qui nous a été suggérée par Emmanuel Lesigne.

Lemme 8. Soit $T$ une transformation d'un espace de probabilité $(M, m)$ et soit $H: M \rightarrow \mathbb{R}$ une fonction m-mesurable. Supposons que le cobord $h=H \circ T-H$ a une partie négative intégrable; alors $h$ est intégrable et $\int_{M} h d m=0$.

Démonstration. Supposons que la mesure $m$ est ergodique. Par hypothèse, $\int_{M} h^{-}(v) d m(v)<\infty$; donc, d'après le théorème ergodique, $\left(\frac{1}{N} \sum_{0}^{N-1} h^{-} \circ T^{k}(v)\right) \rightarrow \int_{M} h^{-}(v) d m(v)$ quand $n \rightarrow \infty$, pour $m$-presque tout $v$. Si $h^{+}$n'est pas intégrable, $\int_{M} h^{+}(v) d m(v)=\infty$; alors, toujours d'après le théorème ergodique, pour $m$-presque tout $v$, la suite $\left(\frac{1}{N} \sum_{0}^{N-1} h^{+} \circ T^{k}(v)\right) \rightarrow \infty$ lorsque $N \rightarrow \infty$. Donc $\left(\frac{1}{N} \sum_{0}^{N-1} h \circ T^{k}(v)\right) \rightarrow \infty$ pour $m$-presque tout $v$, c'est-à-dire : $\lim \frac{1}{N}\left(H \circ T^{N}(v)-H(v)\right)=\infty$, pour $m$-presque tout $v$. Or, comme $H$ est à valeurs finies sur un ensemble de mesure pleine, $H / N$ converge vers 0 en probabilité ; et il en est de même pour $H \circ T^{N} / N$, la mesure $m$ étant $T$-invariante. Donc $h$ est intégrable. Par le théorème ergodique, ses sommes de Birkhoff tendent alors vers $\int_{M} h$, $m$-presque partout. Comme nous l'avons vu, ces sommes tendent vers 0 en probabilité. On a donc: $\int_{M} h d m=0$.

Lorsque $m$ n'est pas ergodique, on note $\mathcal{I}$ la tribu des ensembles $T$-invariants. Puisque $h$ est intégrable, on a encore que $\left(\frac{1}{N} \sum_{0}^{N-1} h \circ T^{k}(v)\right)$ converge presque partout vers $\mathbb{E}(h / \mathcal{I})$, à valeurs dans $]-\infty, \infty]$. Si $h^{+}$n'est pas intégrable, $\mathbb{E}(h / \mathcal{I})$ est d'espérance infinie ; en particulier, $\lim \left(\frac{1}{N} \sum_{0}^{N-1} h \circ T^{k}(v)\right)$ est supérieur à 1 sur un ensemble de mesure $>0$, ce qui contredit que $\frac{1}{N}\left(H \circ T^{N}-H\right)$ tend vers 0 en probabilité. Donc $h$ est intégrable; de plus $\int h d m=0$, car sinon on aboutirait à la même contradiction. 
Une application du Lemme 8 lorsque $m=\mu, T=\phi$ et $H=-F$ donne :

$$
-\int \log \mu_{\xi(v)}\left(\phi^{-1} \xi(v)\right) d \mu(v)=\delta(\Gamma) \tau
$$

Donc, d'après la Proposition $4, h_{\mu}\left(\phi_{\tau}\right)=\delta(\Gamma) \tau$. L'entropie de $\mu$ pour le flot $\left(\phi_{t}\right)$ est donc bien égale à $\delta(\Gamma)$.

Supposons maintenant que $m$ est une mesure de probabilité finie, invariante par le flot $\left(\phi_{t}\right)$ et d'entropie $h_{m}$; on veut montrer que $h_{m} \leq \delta(\Gamma)$ et que l'égalité $h_{m}=\delta(\Gamma)$ entraîne que $m=\mu$, lorsque $\mu$ est finie et aboutit à une contradiction lorsque $\mu$ est infinie. Pour cela, on ne perd rien à supposer que $m$ est ergodique. Choisissons, comme précédemment, un temps $\tau>0$ tel que $m$ est ergodique pour $\phi_{\tau}$ et une partition $m$-mesurable $\xi$ vérifiant les conclusions de la Proposition 1. D'après la Proposition $4, h_{m}\left(\phi_{\tau}\right)=\tau h_{m}$ est égale à $H_{m}\left(\phi_{\tau}^{-1} \xi \mid \xi\right)$.

Nous allons montrer dans un premier temps que les conditionnelles de $m$ et de $\mu$ sont égales pour presque tout atome de $\xi$. Puisque $\xi$ est $m$-mesurable, les conditionnelles de $m$ sur les atomes de $\xi(v)$ sont bien définies pour $m$-presque tout $v$. Il n'en est pas de même pour $\mu$, que nous ne supposons même pas finie pour le moment; toutefois la structure produit de $\mu$ permet de définir sur $m$-presque tout atome $\xi(v)$ une mesure. En effet pour $m$-presque tout $v, \xi(v)$ est un voisinage de $v$ par construction; en particulier sa $\mu_{v}^{+}$-mesure est non-nulle et on peut alors définir une mesure, que nous noterons encore $\mu_{\xi(v)}$, en posant pour tout borélien $A \subset W_{v}^{+}$:

$$
\mu_{\xi(v)}(A)=\frac{\mu_{v}^{+}(A \cap \xi(v))}{\mu_{v}^{+}(\xi(v))} .
$$

Lorsque $\mu$ est une mesure finie, et lorsque $\xi$ est subordonnée à $\mu$, c'est exactement la mesure $\mu_{\xi(v)}$ d'après la fin de la section 2.

La partition $\xi$ étant décroissante et subordonnée au feuilletage instable, $\phi^{-1} \xi \mid \xi(v)$ est une partition de l'atome $\xi(v)$. Notons que, pour $\bar{m}$-presque tout atome $\xi(v)$, la partition $\phi^{-1} \xi \mid \xi(v)$ est au plus dénombrable. On définit pour $m$-presque tout $v$

$$
\psi(v)=\frac{\mu_{\xi(v)}\left(\phi^{-1} \xi(v)\right)}{m_{\xi(v)}\left(\phi^{-1} \xi(v)\right)} .
$$

Fait 9. La fonction $\psi$ définie ci-dessus est m-mesurable. Les fonctions $\psi$ et $\log \psi$ sont $m$ intégrables.

Démonstration. Pour voir que $\psi$ est $m$-mesurable, il suffit de montrer que $\mu_{\xi(v)}\left(\phi^{-1} \xi(v)\right)$ est $m$ mesurable, puisque la mesurabilité du dénominateur découle de la $m$-mesurabilité de $\xi$. Montrons que $v \rightarrow \mu_{v}^{+}(\xi(v))$ est $m$-mesurable, la démonstration pour $v \rightarrow \mu_{v}^{+}\left(\phi^{-1} \xi(v)\right)$ étant identique. La partition $\xi$ est la borne supérieure d'une suite croissante de partitions finies $\xi_{n}$ dont les atomes sont des boréliens : de plus, pour $m$-presque tout $v$, l'atome $\xi_{n}(v)$ est relativement compact lorsque $n$ est assez grand si bien que $\mu_{v}^{+}\left(\xi_{n}(v)\right)$ converge vers $\mu_{v}^{+}(\xi(v)), m$-presque partout. Il suffit donc de montrer que $v \rightarrow \mu_{v}^{+}\left(\xi_{n}(v)\right)$ est $m$-mesurable. Puisque la partition $\xi_{n}$ est finie, il suffit en fait de vérifier que pour tout borélien $C$ de $T^{1} X$, la fonction $v \rightarrow \mu_{v}^{+}(C)$ est $m$-mesurable. Or on a déjà remarqué lorsqu'on a décrit les mesures $\mu_{v}^{+}$que la fonction $v \rightarrow \mu_{v}^{+}(C)$ est borélienne; elle est donc $m$-mesurable.

Pour montrer maintenant que la fonction (positive) $\psi$ est $m$-intégrable, il nous faut voir, d'après la définition de la mesure conditionnelle, que sur l'espace quotient $M_{\xi}$, la fonction mesurable 
$\xi(v) \rightarrow \int_{\xi(v)} \psi(w) d m_{\xi(v)}(w)$ est $\bar{m}$-intégrable. Or, d'après la définition de la mesure conditionnelle, $\int_{\xi(v)} \psi(w) d m_{\xi(v)}(w)=\sum \mu_{\xi(v)}\left(\phi^{-1} \xi^{\prime}\right)$, la somme étant prise sur les atomes de $\phi^{-1} \xi$ (en quantité dénombrable) qui sont contenus dans $\xi(v)$; cette somme est évidemment majorée par $\mu_{\xi(v)}(\xi(v))=$ 1 et $\psi$ est bien $m$-intégrable. On a de plus : $\int_{M} \psi(v) d m(v) \leq 1$.

Pour voir finalement que $\log \psi$ est intégrable, on remarque que $\log \psi$ est la différence des deux fonctions mesurables négatives $\log \mu_{\xi(v)}\left(\phi^{-1} \xi(v)\right)$ et $\log m_{\xi(v)}\left(\phi^{-1} \xi(v)\right)$. L'intégrabilité de la première fonction découle de l'expression $-\log \mu_{\xi(v)}\left(\phi^{-1} \xi(v)\right)=\delta(\Gamma) \tau+F(v)-F(\phi v)$ et du Lemme 8; l'intégrabilité de la deuxième de la Proposition 4 et de ce que l'entropie de $\phi_{\tau}$ est finie.

Nous allons maintenant appliquer l'inégalité de Jensen. Par définition de $\psi$, on a $\int_{M} \psi(v) d m(v) \leq$ 1 ; de plus $\int_{M} \log \psi(v) d m(v)=-\delta(\Gamma) \tau+H_{m}\left(\phi^{-1} \xi \mid \xi\right)$. D'après Jensen, on a donc : $H_{m}\left(\phi^{-1} \xi \mid \xi\right)-\delta(\Gamma) \tau \leq \log \left(\int_{M} \psi(v) d m(v)\right) \leq 0$, d'où l'inégalité : $h_{m} \leq \delta(\Gamma)$.

L'hypothèse $h_{m}=\delta(\Gamma)$ et le cas d'égalité dans l'inégalité de Jensen entraînent alors que $\psi(v)=1$ $m$-presque partout. On en déduit que pour $m$-presque tout $v$, les mesures conditionnelles $m_{\xi(v)}$ et $\mu_{\xi(v)}$ coïncident sur la tribu engendrée $\operatorname{par} \phi^{-1} \xi \mid \xi(v)$. De même, en remplaçant $\phi$ par $\phi^{k}$, on trouve que pour $m$-presque tout $v, m_{\xi(v)}$ et $\mu_{\xi(v)}$ coïncident sur la tribu engendrée par $\phi^{-k} \xi \mid \xi(v)$ pour tout $k \geq 1$. Puisque $\xi$ est génératrice (cf. Proposition 1), les mesures $m_{\xi(v)}$ et $\mu_{\xi(v)}$ sont donc égales, pour $m$-presque tout $v$.

L'argument de Hopf va nous permettre de conclure à présent que $m$ est nécessairement la mesure de Patterson-Sullivan $\mu$. Fixons une fonction $f$ continue et à support compact sur $T^{1} X$. Supposons dans un premier temps que $\mu$ est une mesure finie. Alors $\mu$ est ergodique d'après le théorème de Hopf-Tsuji-Sullivan [Su1]. Donc l'ensemble $\Lambda_{f}$ des vecteurs $v \in \Lambda(\Gamma)$ tels que $\lim _{t \rightarrow \infty} \frac{1}{t} \int_{0}^{t} f\left(\phi_{s} v\right) d s=\mu(f)$ est de $\mu$-mesure pleine; de plus, par l'uniforme continuité de $f, \Lambda_{f}$ est saturé par le feuilletage faiblement stable. Donc, pour toute feuille instable $W_{v}^{+}, \Lambda_{f}$ est de $\mu_{v}^{+}$-mesure pleine; en particulier, pour tout atome $\xi(v)$, l'intersection $\Lambda_{f} \cap \xi(v)$ est de $\mu_{\xi(v) \text {-mesure }}$ pleine. Donc, d'après le paragraphe précédent, $\Lambda_{f}^{\mu} \cap \xi(v)$ est de $m_{\xi(v)}$-mesure pleine pour $\bar{m}$-presque tout atome $\xi(v)$; autrement dit, $\Lambda_{f}$ est de $m$-mesure pleine. Le Théorème ergodique appliqué cette fois à la mesure $m$ donne alors $m(f)=\mu(f)$. La fonction $f$ étant quelconque, il vient $m=\mu$.

Supposons maintenant que $\mu$ est une mesure infinie. Par le théorème de Hopf-Tsuji-Sullivan, ou bien $\Gamma$ est convergent, auquel cas le flot $\left(\phi_{t}\right)$ est complètement dissipatif pour la mesure $\mu$, ou bien $\Gamma$ est divergent, auquel cas $\left(\phi_{t}\right)$ est ergodique et complètement conservatif (pour la mesure $\mu$ ).

Dans le premier cas, pour $\mu$-presque tout $v$, on a $\lim _{t \rightarrow \infty} f\left(\phi_{t} v\right)=0$, et donc aussi $\lim _{t \rightarrow \infty} \frac{1}{t} \int_{0}^{t} f\left(\phi_{s} v\right) d s=0$. Par l'argument du paragraphe précédent, il vient $m(f)=0$ pour tout fonction continue à support compact, ce qui est impossible.

Dans le deuxième cas, il existe sur $T^{1} X$ une fonction $\rho$ strictement positive, continue, bornée et $\mu$-intégrable telle que pour $\mu$-presque tout $v \lim _{t \rightarrow \infty} \int_{0}^{t} \rho\left(\phi_{s} v\right) d s=\infty$ (la construction donnée dans [Su1] en courbure constante s'adapte immédiatement au cas où la courbure est variable). Le Théorème ergodique de Hopf en mesure infinie appliqué à la mesure $\mu$, le Théorème ergodique classique appliqué à $m$ donnent, en reprenant encore l'argument précédent :

$$
\frac{m(f)}{m(\rho)}=\frac{\mu(f)}{\mu(\rho)}
$$

Ceci n'est possible que si les mesures $m$ et $\mu$ sont proportionnelles, alors que nous avions supposé l'une finie, l'autre infinie. Cette contradiction termine la démonstration du Théorème 2. 


\section{$\S 4$. Entropie topologique.}

Avant de démontrer le Théorème 1, nous allons maintenant rappeler la définition de Bowen de l'entropie topologique d'un flot $\left(\phi_{t}\right)$ agissant sur un espace métrique non-compact $(E, d)$ (cf. [Bowe]). Pour tout $T>0$, on note $d_{T}$ la distance sur $E$ définie par

$$
d_{T}(x, y)=\sup _{0 \leq t \leq T} d\left(\phi_{t}(x), \phi_{t}(y)\right)
$$

Soit $\epsilon>0$ et soit $K \subset E$ un compact. Un ensemble $D \subset K$ est $(K, T, \epsilon)$-dense si pour tout point $x \in K$, il existe $y \in D$ tel que $d_{T}(x, y) \leq \epsilon$. Un ensemble $S \subset K$ est $(K, T, \epsilon)$-séparé si pour tous points distincts $x, y$ de $S, d_{T}(x, y) \geq \epsilon$. On définit alors $h_{d}$, l'entropie de la distance $d$ en posant:

$$
h_{d}=\sup _{K} \sup _{\epsilon>0} \limsup _{T \rightarrow \infty} \frac{1}{T} \log d(K, T, \epsilon)=\sup _{K} \sup _{\epsilon>0} \limsup _{T \rightarrow \infty} \frac{1}{T} \log s(K, T, \epsilon)
$$

où $d(K, T, \epsilon)$ est le cardinal minimum d'un ensemble $(K, T, \epsilon)$-dense et $s(K, T, \epsilon)$ le cardinal maximum d'un ensemble $(K, T, \epsilon)$-séparé.

Contrairement au cas d'un flot sur un espace compact, cette définition ne dépend pas uniquement de la topologie sur l'espace. On définit l'entropie topologique $h_{\text {top }}$ comme l'infimum des entropies $h_{d}$, lorsque $d$ décrit l'ensemble des distances sur $E$, qui définissent la topologie de $E$.

Dans le cas du flot géodésique sur $\Lambda(\Gamma)$, la distance naturelle est la distance riemannienne dont nous donnons une définition. Un vecteur $v \in T^{1} X$ détermine une géodésique $v_{s}: \mathbb{R} \rightarrow X$ de condition initiale $v_{0}=v$. Si on munit $T^{1} X$ de la distance $d\left(v, v^{\prime}\right)=\sup _{s \in[-1,1]} d_{X}\left(v_{s}, v_{s}^{\prime}\right)$, où $d_{X}$ est la distance riemannienne sur $X$, la distance est équivalente à la distance riemannienne sur $T^{1} X$. On définit de la même façon la distance $\tilde{d}$ sur $T^{1} \tilde{X}$.

Démonstration du Théorème 1. Comme il a été dit dans l'introduction, il suffit de montrer que pour toute distance $d^{\prime}$ sur $\Lambda(\Gamma)$, on a : $h_{d^{\prime}} \geq \delta(\Gamma)$. Nous allons d'abord considérer le cas de la distance riemannienne $d$ ci-dessus; à la fin, nous indiquerons comment modifier l'argument pour une distance $d^{\prime}$ quelconque. Nous allons construire, pour tout $\delta^{\prime}<\delta(\Gamma)$ un compact $K \subset \Lambda$ et une suite d'ensembles $\left(K, T_{i}, \epsilon\right)$-séparés de cardinal $\geq C e^{\delta^{\prime} T_{i}}$, pour une suite $\left(T_{i}\right)$ tendant vers $\infty$, une constante $C>0$ indépendante de $\left(T_{i}\right)$, et un certain $\epsilon>0$. Nous ne ferons qu'adapter un argument d'un exposé d'Ursula Hamenstädt (cf. [H]) qui portait sur le théorème de Bishop-Jones [BJ].

Si $B(x, C)$ est la boule de rayon $C$ centrée au point $x \in \tilde{X}$, on note $\mathcal{O}(x, C)$ son ombre vue de l'origine $o$, c'est-à-dire l'ensemble des extrémités dans $\partial \tilde{X}$ des rayons géodésiques issus de $o$ et passant par un point de $B(x, C)$.

Le résultat suivant est une conséquence de l'inégalité triangulaire pour (1), des théorèmes de comparaison pour (2).

\section{Fait 10.}

(1) Pour tout $C>0$, si $\left|d_{\tilde{X}}(o, x)-d_{\tilde{X}}(o, y)\right| \leq 1$ et $d_{\tilde{X}}(x, y) \geq 4 C+1$, alors les ombres $\mathcal{O}(x, C)$ et $\mathcal{O}(y, C)$ sont disjointes;

(2) Il existe une constante $C_{0}>0$ telle que pour tout $C \geq C_{0}$, pour tout angle $\theta \leq \pi / 4$, il existe $R(C)$ telle que si deux points $x$ et y sont à distance supérieure à $R(C)$, et si l'angle $\widehat{o x y}$ du triangle géodésique oxy est supérieur à $\pi-\theta$, alors $\mathcal{O}(y, C) \subset \mathcal{O}(x, C)$.

Rappelons finalement la propriété suivante des quasi-géodésiques dans une variété à courbure négative. Il existe une constante $\eta(\theta)$ qui tend vers 0 avec $\theta$ telle que : tout chemin $c$ réunion de 
segments géodésiques faisant des angles supérieurs à $\pi-\theta$, est contenu dans le voisinage tubulaire de rayon $\eta(\theta)$ de la géodésique qui a les mêmes extrémités, dès lors que les longueurs des segments géodésiques sont toutes supérieures à une constante $R(\theta)$.

Fixons $0<\theta \leq \pi / 4$ et $R \geq \max \left(R\left(C_{0}\right), R(\theta)\right)$; sans perte de généralité, on peut supposer que $\eta(\theta)$ est inférieur au rayon d'injectivité en la projection de $o$ sur $X$. Soit $\mathcal{G} \subset \Gamma$ un ensemble fini d'éléments tels que :

(1) $\left|d_{\tilde{X}}(o, g o)-R\right| \leq 1 / 2$,

(2) $d_{\tilde{X}}\left(g o, g^{\prime} o\right) \geq 4 C_{0}+1$ pour tous les éléments distincts $g, g^{\prime}$, et

(3) il existe un vecteur $v \in T^{1} X$ tel que pour tout $g \in \mathcal{G}$, le segment géodésique $[o, g o]$ se projette sur $X$ en un lacet dont les vecteurs tangents en ses extrémités font un angle inférieur à $\theta / 2$ avec $v$.

Pour tout entier $k>0$, considérons l'ensemble $\mathcal{G}^{k}$ des mots de longueur $k$ dans le semi-groupe engendré par $\mathcal{G}$. En raisonnant par récurrence sur $k$, on voit que le Fait 10 et les propriétés vérifiées par l'ensemble $\mathcal{G}$ entraînent d'une part que le cardinal de $\mathcal{G}^{k}$ est $(\sharp \mathcal{G})^{k}$, et même que, si $g$ et $g^{\prime}$ sont deux mots distincts de $\mathcal{G}^{k}$, les ombres $\mathcal{O}(g o, C)$ et $\mathcal{O}\left(g^{\prime} o, C\right)$ sont disjointes.

Alors si $g=g_{i_{1}} \ldots g_{i_{k}} \in \mathcal{G}^{k}$, le segment géodésique $[o, g o]$ se projette sur $X$ en un lacet $l_{g}$ librement homotope à une géodésique fermée $c_{g}$ par une homotopie qui bouge les points de moins que $\eta(\theta)$ : notons $v^{g}$ le vecteur unitaire tangent à $c_{g}$, au point image de $o$ par cette homotopie. Soit $K \subset T^{1} X$ le compact défini comme le $\eta(\theta)$-voisinage de l'ensemble des vecteurs tangents aux géodésiques $l_{g}$, où $g \in \mathcal{G}$. Soit $\epsilon>0$ une constante strictement inférieure d'une part à $2 C-2 \eta(\theta)$, d'autre part au rayon d'injectivité du compact $\pi(K)$.

Fait 11. Pour tout entier $k$, l'ensemble des vecteurs $v^{g}$ lorsque $g$ décrit $\mathcal{G}^{k}$ est $(K, k(R+1 / 2), \epsilon)$ séparés.

Démonstration. La propriété de $\eta(\theta)$ entraîne que les orbites par le flot géodésique des vecteurs $v^{g}$, (c'est-à-dire l'ensemble des vecteurs tangents aux géodésiques $c_{g}$ ) sont contenues dans $K$; d'autre part, puisque $\eta(\theta)$ est inférieur au rayon d'injectivité de $X$ en le projeté de $o$, chaque vecteur $v^{g}$ a un unique relevé $\tilde{v}^{g}$, basé en un point à distance inférieure à $\eta(\theta)$ de $o$, et la géodésique qu'il détermine est à distance inférieure à $\eta(\theta)$ du segment $[o, g o]$. Donc pour deux mots différents $g$ et $g^{\prime}$ de $\mathcal{G}^{k}$, il existe un premier temps $\tau \leq k(R+1 / 2)$ où les points $\tilde{v}_{\tau}^{g}$ et $\tilde{v}_{\tau}^{g^{\prime}}$ sont à distance supérieure à $\epsilon$ (puisque $\tilde{v}_{k(R+1 / 2)}^{g}$ et $\tilde{v}_{k(R+1 / 2)}^{g^{\prime}}$ sont à distance supérieure à $\left.2 C-2 \eta(\theta)\right)$. Il en est de même alors pour les points $v_{\tau}^{g}$ et $v_{\tau}^{g^{\prime}}$ d'après le choix de $\epsilon$.

D'après ce qui précède, s'il existe un point $o \in \tilde{X}$ et un ensemble $\mathcal{G}$ vérifiant (1), (2) et (3) de cardinal $\sharp \mathcal{G} \geq e^{\delta^{\prime}(R+1 / 2)}$, alors on aura : $h_{d} \geq \delta^{\prime}$. Dans le but de construire ce point et cet ensemble, rappelons d'abord que pour tout groupe Kleinien $\Gamma$ d'isométries de $\tilde{X}$, le flot géodésique $\left(\phi_{t}\right)$ restreint à l'ensemble non-errant $\Lambda(\Gamma)$ admet un vecteur $u$ d'orbite dense [Eb] ; soit $u$ un tel vecteur, basé en un point noté $o \in \tilde{X}$. La divergence de la série de Poincaré pour tout exposant $\delta^{\prime}<\delta(\Gamma)$ entraîne l'existence, pour toute constante $C>0$, d'une suite $\left(R_{i}\right)$ tendant vers l'infini telle que l'ensemble des éléments $\gamma \in \Gamma$ vérifiant $d_{\tilde{X}}(o, \gamma o) \simeq R_{i} \pm 1 / 4$ a un cardinal supérieur à $C e^{\delta^{\prime} R_{i}}$. Soit $0<\theta<\pi / 4$. La compacité de la sphère unité du fibré tangent au point $o$ et la discrétude de l'orbite $\Gamma o$ entraînent alors qu'il existe deux vecteurs $v$ et $w$ dans cette sphère unité tels que : pour tout angle $\theta>0$, il existe une suite $\left(R_{i}\right)$ tendant vers $\infty$ et des ensembles $\mathcal{G}\left(R_{i}, \theta\right) \subset \Gamma$ de cardinal supérieur à $e^{\delta^{\prime} R_{i}}$ dont les éléments $\gamma, \gamma^{\prime}$ vérifient :

(1) $d_{\tilde{X}}(o, \gamma o) \simeq R_{i} \pm 1 / 4$

(2) $d_{\tilde{X}}\left(\gamma o, \gamma^{\prime} o\right) \geq 4 C_{0}+1$ 
(3) les vecteurs tangents au point $o$ (resp. $\gamma o$ ) aux segments géodésiques $[o, \gamma o]$ se projettent en des vecteurs situés dans le cône d'amplitude $\theta / 4$ autour de $v$ (resp. autour de $w$ ).

Alors si $v=w$, pour tout angle $\theta \leq \pi / 4$, le point $o$ et l'ensemble $\mathcal{G}\left(R_{i}, \theta\right)$ auront les propriétés voulues pour $i$ suffisamment grand.

Lorsque $v \neq w$, on distingue deux cas :

1) $v=-w$. Supposons dans un premier temps $v$ proche de $u$ (le cas où $v$ est proche de $-u$ se traîte de façon analogue). Puisque l'orbite de $-u$ est dense, il existe un lacet géodésique basé en $o$ dont le vecteur tangent est proche de $-u$ en son origine et proche de $u$ en son extrémité ; en outre, plus la longueur de ce lacet est grande, plus ces vecteurs peuvent être rendus proches de $-u$ et $u$ respectivement. Soit $a$ l'élément de $\Gamma$ représentant la classe d'homotopie d'un tel lacet. Alors le point $o$ et l'ensemble des éléments $a \gamma$ pour $\gamma \in \mathcal{G}\left(R_{i}, \theta\right)$ auront les propriétés voulues si $\theta$ est assez petit et $i$ assez grand.

Supposons maintenant que $v$ fait un angle $\varphi>0$ avec $u$ et avec $-u$. Par la densité de l'orbite de $u$, il existe des lacets géodésiques basés en $o$ dont les vecteurs tangents en $o$ sont arbitrairement proches de $u$, faisant par exemple un angle inférieur à $\varphi / 4$. Soit $a \in \Gamma$ un élément représentant la classe d'homotopie d'une telle géodésique. Alors le point $o$ et les éléments $a \gamma a$, pour $\gamma \in \mathcal{G}\left(R_{i}, \varphi / 4\right)$, auront les propriétés voulues dès que la longueur de $a$ est assez grande, pour tout $i$ assez grand.

2) $v \neq-w$. Dans le triangle isocèle $o p q$ dont les côtés en $o$ sont les segments géodésiques déterminés par les vecteurs $v$ et $w$ de longueur $\rho$, les angles aux sommets $p$ et $q$ tendent vers 0 lorsque $\rho$ tend vers $\infty$. Notons $a$ le segment géodésique $[q, o]$ et $\mathcal{G}^{\prime}\left(R_{i}, \theta\right)$ l'ensemble des éléments de $\Gamma$ représentant les lacets $a^{-1} \gamma a$ basés en $q$, où $\gamma \in \mathcal{G}\left(R_{i}, \theta\right)$. Alors pour $\rho$ assez grand, le point $q$ et l'ensemble $\mathcal{G}^{\prime}\left(R_{i}, \theta\right)$ auront d'après le cas 1 les propriétés voulues si $\theta$ est assez petit et $i$ assez grand.

Soulignons que pour tout élément $g$ dans le semi-groupe engendré par $\mathcal{G}$, l'orbite du vecteur $v_{g}$ reste dans $K$. Si $d^{\prime}$ est une distance topologiquement équivalente à $d$, sa restriction à $K$ appartient à la même classe uniforme que celle de $d$. Ainsi, il existe $\epsilon^{\prime}>0$ tel que l'ensemble des vecteurs $v^{g}$ du Fait 11 soit $\left(K, k(R+1 / 2), \epsilon^{\prime}\right)$-séparé pour $d^{\prime}$. On aura donc $h_{d^{\prime}} \geq \delta(\Gamma)$.

Démonstration de la Proposition 3. Nous sommes maintenant dans la situation d'un groupe $\Gamma$ géométriquement fini et nous garderons les mêmes notations pour ces groupes que celles du $\S 1$.

Par le Théorème 1, il nous suffit de montrer que pour la distance $d$, on a : $h_{d} \leq \delta(\Gamma)$. Fixons un compact $K \subset \Lambda(\Gamma)$ et une constante $\delta^{\prime}>\delta(\Gamma)$. Nous allons montrer que pour toute constante $\epsilon$ suffisamment petite, tout ensemble $(K, T, \epsilon)$-séparé $\mathcal{S}$ a un cardinal inférieur à $\kappa T^{2} e^{\delta^{\prime} T}$ où la constante $\kappa$ ne dépend pas de $T$.

Nous supposerons dans la suite que les horoboules $\mathcal{H}_{i}, i=1, \ldots l$ ont été choisies de sorte que, pour tout $r \geq 0$, le diamètre de l'ensemble des vecteurs orthogonaux à $\partial \mathcal{H}_{i} \times\{r\}$ basés en des points de $N_{i}^{r}$ est inférieur à $\epsilon / 4$; ceci est possible, quitte à remplacer $\mathcal{H}_{i}$ par $\partial \mathcal{H}_{i} \times\left[r_{0}, \infty[\right.$ pour $r_{0}$ assez grand, puisque le diamètre de $N_{i}^{r}$ tend vers 0 quand $r \rightarrow \infty$ et que la deuxième forme fondamentale des horosphères est comprise entre $\alpha$ Id et $\beta$ Id. Sans perte de généralité, on peut aussi supposer que la projection $\pi(K)$ est contenue dans le compact $C_{0}$.

Choisissons maintenant un compact $\tilde{C}_{0} \subset \tilde{X}$ qui se surjecte sur $C_{0}$ par la projection de revêtement de $\tilde{X}$ sur $X$.

Si $v \in K$, le segment géodésique $\left(v_{t}\right)_{t \in[0, T]}$ joint un point de $C_{0}$ au point $v_{T}$ qui est contenu soit dans $C_{0}$ soit dans l'un des bouts cuspidaux $C_{i}, i=1, \ldots, l$. On partitionne $\mathcal{S}$ en deux ensembles $\mathcal{S}^{+}$et $\mathcal{S}^{-}$où $v \in \mathcal{S}^{ \pm}$selon que $v_{T}$ est ou n'est pas dans $C_{0}$. On va d'abord montrer que le cardinal 
de $\mathcal{S}^{+}$est inférieur à $c_{+} e^{\delta^{\prime} T}$ puis que celui de $\mathcal{S}^{-}$est inférieur à $c_{-} T^{2} e^{\delta^{\prime} T}$, pour des constantes $c_{-}$ et $c_{+}$indépendantes de $T$.

Pour un vecteur $v \in K$, choisissons un relevé $\tilde{v}$ de $v$ qui est contenu dans $\tilde{C}_{0}$. Alors, si $v_{T} \in C_{0}$, $\tilde{v}_{T}$ appartient à un translaté $\gamma \tilde{C}_{0}$, pour un certain $\gamma \in \Gamma$. Le nombre de translatés $\gamma \tilde{C}_{0}$ dont la distance à $\tilde{C}_{0}$ est inférieure à $T$ est majoré par $c e^{\delta^{\prime} T}$, où, par définition de l'exposant critique, la constante $c$ ne dépend pas de $T$. D'après la convexité de la fonction distance entre deux géodésiques, et puisque la courbure de $\tilde{X}$ est minorée, il existe une constante $\epsilon^{\prime}>0$, indépendante de $T \geq 1$ telle que pour tous vecteurs $\tilde{v}^{1}, \tilde{v}^{2}$ de $T^{1} \tilde{X}$ vérifiant $d_{\tilde{X}}\left(\tilde{v}_{0}^{1}, \tilde{v}_{0}^{2}\right) \leq \epsilon^{\prime}$ et $d_{\tilde{X}}\left(\tilde{v}_{T}^{1}, \tilde{v}_{T}^{2}\right) \leq \epsilon^{\prime}$, on a $\tilde{d}_{T}\left(\tilde{v}^{1}, \tilde{v}^{2}\right) \leq \epsilon$; a fortiori, si $v_{i} \in T^{1} X$ désigne le projeté de $\tilde{v}_{i}$, on a $d_{T}\left(v^{1}, v^{2}\right) \leq \epsilon$ puisque la projection de revêtement décroît les distances. Soit $k$ le cardinal maximal d'un ensemble $\epsilon^{\prime} / 2$ séparé dans $\tilde{C}_{0}$. Le cardinal de l'ensemble des relevés de $S^{+}$appartenant à $\tilde{C}_{0}$ est donc inférieur à $c k^{2} e^{\delta^{\prime} T}$; il en est de même pour le cardinal de $S^{+}$.

Afin de majorer le cardinal de $\mathcal{S}^{-}$, nous allons d'abord étudier la "restriction" du flot géodésique à la préimage des pointes, c'est-à-dire à $\pi^{-1}\left(C_{i}\right) \cap \Lambda(\Gamma), i \geq 1$. Fixons $1 \leq i \leq l$ et considérons les vecteurs de $\Lambda(\Gamma)$, basés en des points de $\partial \mathcal{H}_{i}$ et qui pointent dans l'horoboule $\mathcal{H}_{i}$; pour $t>0$; notons $W_{t}$ l'ensemble de ces vecteurs $w$ tels que le segment géodésique $\left\{w_{s}\right\}_{0 \leq s \leq t}$ reste contenu dans $\mathcal{H}_{i}$.

Lemme 12. Il existe une constante $c^{\prime}$ telle que tout ensemble $(t, \epsilon)$-séparé de vecteurs contenus dans $W_{t}$ a un cardinal inférieur à $c^{\prime} t$.

Démonstration. Considérons un vecteur $w \in W_{t}$ et $s \rightarrow w_{s}$ la géodésique qu'il détermine. Soient $\tilde{w}$ un relevé de $w$ basé sur $\partial \tilde{\mathcal{H}}_{i}$, et $s \rightarrow \tilde{w}_{s}$ la géodésique qu'il détermine. Soit $s \rightarrow h_{w}(s)$ (resp. $s \rightarrow h_{\tilde{w}}(s)$ ) la fonction égale à la distance de $w_{s}$ à l'horosphère $\partial \mathcal{H}_{i}\left(\right.$ resp. $\left.\partial \tilde{\mathcal{H}}_{i}\right)$; on a $h_{w}=h_{\tilde{w}}$. D'autre part, $h_{w}$ est une fonction strictement concave inférieure à $s$; en particulier, elle atteint son maximum sur l'intervalle $[0, t]$ en un point $s_{0}$. Notons $\tilde{w}^{\prime}$ le vecteur unitaire basé en $\tilde{w}_{0}$ orthogonal à l'horosphère contenant ce point (c'est-à-dire $\partial \tilde{\mathcal{H}}_{i}$ ) et pointé vers le centre de $\tilde{\mathcal{H}}_{i}$. Lorsque $t>s_{0}$, notons $\tilde{w}^{\prime \prime}$ le vecteur tel que $\phi_{t}\left(\tilde{w}^{\prime \prime}\right)$ soit le vecteur unitaire basé en $\tilde{w}_{t}$, orthogonal à l'horosphère contenant $\tilde{w}_{t}$ (c'est-à-dire $\left.\partial \tilde{\mathcal{H}}_{i} \times\left\{h_{\tilde{w}}(t)\right\}\right)$ et orienté vers l'extérieur de $\tilde{\mathcal{H}}_{i}$. Le résultat suivant compare les trajectoires des vecteurs $\tilde{w}$ et $\tilde{w}^{\prime}, \tilde{w}^{\prime \prime}$.

Fait 13. Il existe une constante $c^{\prime}>0$, indépendante de $w$ et de $t$ telle que :

(1) $d\left(\phi_{s}(\tilde{w}), \phi_{s}\left(\tilde{w}^{\prime}\right)\right) \leq c^{\prime} e^{-\beta\left(s_{0}-s\right)}$, pour $s \leq s_{0}$,

(2) $d\left(\phi_{s}(\tilde{w}), \phi_{s}\left(\tilde{w}^{\prime \prime}\right)\right) \leq c^{\prime} e^{-\beta\left(s-s_{0}\right)}$, pour $s_{0}<s \leq t$.

Démonstration. Par définition de $s_{0}$, les points $\tilde{w}_{s_{0}}$ et $\tilde{w}_{h\left(s_{0}\right)}^{\prime}$ sont sur la même horosphère; il est facile de voir que leur distance est bornée indépendamment de $w$. Supposons d'abord $s \leq s_{0}$. D'après les théorèmes de comparaison $[\mathrm{CE}] d_{\tilde{X}}\left(\tilde{w}_{s}, \tilde{w}_{s}^{\prime}\right) \leq c_{1} e^{-\beta\left(s_{0}-s\right)}$ et l'angle entre $\phi_{s}(\tilde{w})$ et le vecteur normal rentrant à l'horosphère passant par $\tilde{w}_{s}$ est inférieur à $c_{2} e^{-\beta\left(s_{0}-s\right)}$; ces inégalités entraînent le résultat cherché. Quand $s \geq s_{0}$, on se ramène au cas précédent par symétrie.

La signification géométrique de ce fait est que l'orbite $\left\{\phi_{s}(w)\right\}_{0 \leq s \leq t}$ du vecteur $w \in W_{t}$ est "essentiellement" déterminée par le point $s_{0}: \phi_{s_{0}}(\tilde{w})$ est sur une horosphère à hauteur $\simeq s_{0}, \phi_{t}(\tilde{w})$ est sur une horosphère à hauteur $\simeq 2 s_{0}-t$, et il existe $l(\epsilon)>0$ tel que hors d'un intervalle de longueur $2 l(\epsilon)$ centré en $s_{0}$, l'orbite de $w$ reste $\epsilon / 4$-proche de celle d'un vecteur orthogonal à $\partial \mathcal{H}_{i}$ : sur l'intervale $0 \leq s \leq s_{0}-l(\epsilon)$, c'est le vecteur $\tilde{w}^{\prime}$, sur l'intervalle $s_{0}+l(\epsilon) \leq s \leq t$, c'est le vecteur $\tilde{w}^{\prime \prime}$. Puisque la projection de revêtement $T^{1} \tilde{X} \rightarrow T^{1} X$ décroît les distances, on a la même description pour l'orbite de $\left\{\phi_{s}(w)\right\}_{0 \leq s \leq t}$ : si $w^{\prime}$ et $w^{\prime \prime}$ sont les projections dans $T^{1}(X)$ des vecteurs $\tilde{w}^{\prime}$ et $\tilde{w}^{\prime \prime}$, alors l'orbite $\left\{\phi_{s}(w)\right\}$ est $\epsilon / 4$-proche de celle du vecteur $w^{\prime}$ pour $0 \leq s \leq s_{0}-l(\epsilon)$, et de 
celle de $w^{\prime \prime}$ pour $s_{0}+l(\epsilon) \leq s \leq t$. Mais d'après le choix des horosphères, le diamètre des vecteurs normaux à $N_{i}^{r}$ est inférieur à $\epsilon / 4$. Donc si $w^{1}$ et $w^{2}$ sont deux vecteurs de $W_{t}$ tels que les fonctions $h_{w^{1}}$ et $h_{w^{2}}$ atteignent leur maximum en des points $s_{0}^{1}$ et $s_{0}^{2}$ différents de moins que $\epsilon / 8$ et tels que les horosphères contenant $w_{t}^{1}$ et $w_{t}^{2}$ sont à distance $\leq \epsilon / 8$, alors la distance entre les orbites $\phi_{s}\left(w^{1}\right)$ et $\phi_{2}\left(w^{2}\right)$ est inférieure à $\epsilon$ sur les intervalles $\left[0, s_{0}^{1}-l(\epsilon)\right]$ et $\left[s_{0}^{1}+l(\epsilon), t\right]$.

Reprenons la démonstration du Lemme 12. Soit $S$ un ensemble $(t, \epsilon)$-séparé contenu dans $W_{t}$. Partitionnons $S$ en sous-ensembles $S_{n, m}$, de sorte que pour $w \in S_{n, m}$, la fonction $h_{w}$ atteigne son maximum en un point de l'intervalle $\left[n \epsilon / 8,(n+1) \epsilon / 8\right.$ [ et que $h_{w}(t)$ appartienne à $[m \epsilon / 8,(m+1) \epsilon / 8[$. D'après le paragraphe précédent, les instants où les orbites de deux vecteurs distincts de $S_{n, m}$ se séparent appartiennent nécessairement à l'intervalle $\left[n \epsilon / 8-l_{\epsilon}, n \epsilon / 8+l_{\epsilon}\right]$ : le cardinal de $S_{n, m}$ est donc majoré par le cardinal maximal d'un ensemble $\epsilon$-séparé de vecteurs basés dans une boule de rayon $2 l_{\epsilon}$ de $\tilde{X}$. Pour terminer la démonstration du lemme, il suffit de voir que le nombre de classes $S_{n, m}$ est inférieur à $c(\epsilon) t$. Or, si pour un vecteur $w \in W$, le maximum de $h_{w}$ est atteint en un point $s_{0} \leq t$, on a $h_{w}(t) \in\left[2 s_{0}-t-c, 2 s_{0}-t+c\right]$, pour une constante $c$ indépendante de $w$. Le nombre des classes $S_{n, m}$ est donc inférieur à $C(\epsilon) t$.

Terminons la démonstration de la majoration du cardinal de $\mathcal{S}$. Par définition, pour $v \in \mathcal{S}^{-}$, le point $v_{T}$ est contenu dans l'une des horoboules $\mathcal{H}_{i}$; il existe donc $\tau(v) \leq T$ tel que le segment géodésique $\left\{v_{s}\right\}_{0 \leq s \leq T}$ entre une dernière fois dans l'une des horoboules $\mathcal{H}_{i}$ à l'instant $\tau(v)$ et $\mathrm{y}$ reste jusqu'à l'instant $T$. Partitionnons $\mathcal{S}^{-}$en sous-ensembles $\mathcal{S}_{n, i}^{-}$tels que si $v \in \mathcal{S}_{n, i}^{-}$le segment géodésique $\left\{v_{s}\right\}_{0 \leq s \leq T}$ entre dans l'horoboule $\mathcal{H}_{i}$ au temps $\tau(v) \in[n \epsilon / 2,(n+1) \epsilon / 2$ [ et y reste jusqu'à l'instant $T$. Choisissons dans $\mathcal{S}_{n, i}^{-}$un sous-ensemble $\mathcal{S}^{\prime}$ de cardinalité maximale, qui soit $((n+1) \epsilon / 2, \epsilon)$-séparé dans $\mathcal{S}_{n, i}^{-}$; le cardinal de $\mathcal{S}^{\prime}$ est inférieur à $c(\epsilon) e^{\delta^{\prime} n \epsilon / 2}$ d'après la première partie de la démonstration. Par maximalité, tout vecteur de $\mathcal{S}_{n, i}^{-}$est $((n+1) \epsilon / 2, \epsilon)$-proche d'un vecteur de $\mathcal{S}^{\prime}$. Puisque $\mathcal{S}$ est $(T, \epsilon)$-séparé, les vecteurs $\left\{\phi_{\tau(v)}(v)\right\}$, où $v$ décrit l'ensemble des vecteurs proches d'un même élément $v^{\prime}$ de $\mathcal{S}^{\prime}$, forment un ensemble $(T-n \epsilon / 2, \epsilon)$-séparé contenu dans $W_{T-(n+1) \epsilon / 2}$; d'après le Lemme 12 , le cardinal de cette famille est inférieur à $c^{\prime}(T-n \epsilon / 2)$. Finalement, le cardinal de $\mathcal{S}_{n, i}^{-}$est majoré par $c^{\prime}(\epsilon)(T-n \epsilon / 2) e^{\delta^{\prime} n \epsilon / 2}$. Le cardinal de $\mathcal{S}^{-}$est donc

inférieur à $c^{\prime}(\epsilon) \sum_{n \leq 2 T / \epsilon}(T-n \epsilon / 2) e^{\delta^{\prime} n \epsilon / 2}$, somme que l'on peut majorer par $c_{-}(\epsilon) T^{2} e^{\delta^{\prime} T}$. Le cardinal de $\mathcal{S}$ est donc inférieur à $c(\epsilon) T^{2} e^{\delta^{\prime} T}$, ce qui entraîne $h_{d} \leq \delta^{\prime}$. Ceci ayant lieu pour tout $\delta^{\prime}>\delta(\Gamma)$, on a $h_{d} \leq \delta(\Gamma)$

\section{REFERENCES}

[A] A. Ancona, Exemples de surfaces hyperboliques de type divergent, de mesures de Sullivan associées finies mais non géométriquement finies, manuscrit non-publié (1999).

[BJ] C. Bishop, P. Jones, Hausdorff dimension and Kleinian groups, Acta Math. 179 (1997), 1-39.

[Bou] M. Bourdon, Structure conforme au bord et flot géodésique d'un CAT (-1)-espace, Enseign. Math. 41 (1995), 63-102.

[Bowd] B. Bowditch, Geometrical finiteness with variable negative curvature, Duke Math. J. 77 (1995), $229-274$.

[Bowe] R. Bowen, Topological entropy for non-compact sets, Trans. American Math. Soc. 184 (1973), 125-136.

[BD] R. Bowen et D. Ruelle, The ergodic theory of Axiom A flows, Invent. Math. 3 (1975), 181-202.

[CE] J. Cheeger et D. Ebin, Comparison theorems in Riemannian geometry, vol. 9, North-Holland Mathematical Library, 1975.

[CI] K. Corlette et A. Iozzi, Limit sets of isometry groups of exotic hyperbolic spaces, Trans. Amer. Math. Soc. 351 (1999), 1507-1530.

[DOP] F. Dal'bo, J.-P. Otal et M. Peigné, Séries de Poincaré des groupes géométriquement finis, Israel J. Math. 118 (2000), 109-124.

[Eb] P. Eberlein, Geodesic flows on negatively curved manifolds, I., Ann. of Math. 95 (1972), $492-510$. 
[Ha] U. Hamenstädt, Conférence à Orléans au séminaire de Systèmes Dynamiques : "Dimension de Hausdorff de l'ensemble limite radial des groupes Kleiniens" (8 Décembre 1999).

[HK] M. Handel, B. Kitchens, Metrics and entropy for non-compact spaces, Israel J. Math. 91 (1994), $253-271$.

[K1] V. Kaïmanovitch, Invariant measures of the geodesic flow and measures at infinity on negatively curved manifolds, Ann. IHP 53 (1990), 361-393.

[K2] V. Kaïmanovitch, Ergodic properties of the horocycle flow and classification of fuchsian groups, J. Dynam. Control Systems 6 (2000), 21-56.

[LS] F. Ledrappier et Strelcyn, A proof of the estimation from below in Pesin's formula, Ergodic Theory Dynam. Systems 2 (1983), 203-219.

[LY] F. Ledrappier, L.-S. Young, The metric entropy of diffeomorphisms, I, Ann. of Math. 2 (1985), 509-539.

[Pa] S. J. Patterson, The limit set of a Fuchsian group, Acta Math. 136 (1976), 241-273.

[Pe] M. Peigné, On the Patterson-Sullivan measure of some discrete groups of isometries, Israel J. Math. 133 (2003), 77-88.

[Ro] V. A. Rokhlin, Lectures on the entropy theory of measure-preserving transformations, Russian Mathematical Surveys (1967), 1-51.

[Su1] D. Sullivan, The density at infinity of a discrete group of hyperbolic motions, Publ. IHES 50 (1979), $171-202$.

[Su2] D. Sullivan, Entropy, Hausdorff measures old and new, and limit sets of geometrically finite Kleinian groups, Acta Math. 153 (1984), 259-277.

[W] P. Walters, An Introduction to Ergodic Theory, Springer Verlag, 1982.

[Y] C. Yue, The ergodic theory of discrete isometry groups on manifolds of variable negative curvature, Trans. Amer. Math. Soc. 348 (1996), 4965-5005.

Jean-Pierre Otal : jpotal@umpa.ens-lyon.fr

Marc Peigné : peigne@univ-tours.fr 